\title{
Martian low-altitude magnetic topology deduced from MAVEN/SWEA observations
}

Shaosui $\mathrm{Xu}^{1}$, David Mitchell ${ }^{1}$, Michael Liemohn ${ }^{2}$, Xiaohua Fang ${ }^{3}$, Yingjuan Ma ${ }^{4}$, Janet Luhmann $^{1}$, David Brain ${ }^{3}$, Morgane Steckiewicz ${ }^{5}$, Christian Mazelle ${ }^{5}$, Jack Connerney ${ }^{6}$, Bruce Jakosky $^{3}$

${ }^{1}$ Space Science Laboratory, University of California, Berkeley, USA

${ }^{2}$ Department of Climate and Space Sciences and Engineering, University of Michigan, Ann Arbor, USA

${ }^{3}$ Laboratory of Atmospheric and Space Sciences, University of Colorado, Boulder, USA

${ }^{4}$ Department of Earth Planetary and Space Sciences, University of California, Los Angeles, USA

${ }^{5}$ IRAP, CNRS and University Paul Sabatier, Toulouse, France

${ }^{6}$ Goddard Space Flight Center, Greenbelt, Maryland, USA

Corresponding author: Shaosui Xu (shaosui.xu@ssl.berkeley.edu), Space Science Laboratory, University of California, Berkeley, USA

Key Points:

- Pitch angle-resolved electron energy shape parameters are used to deduce magnetic topology

- Closed magnetic field lines dominate low altitudes $(<400 \mathrm{~km})$ of the northern hemisphere on the dayside

- $3 \mathrm{D}$ view of the Martian magnetic topology is presented for the first time

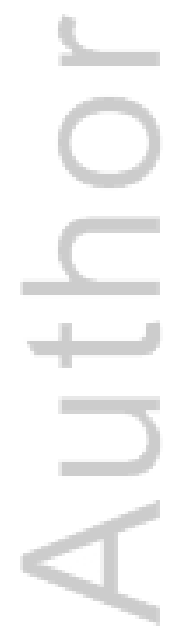

This is the author manuscript accepted for publication and has undergone full peer review but has not been through the copyediting, typesetting, pagination and proofreading process, which may lead to differences between this version and the Version of Record. Please cite this article as doi: $10.1002 / 2016 J A 023467$

This article is protected by copyright. All rights reserved. 


\begin{abstract}
The Mars Atmosphere and Volatile Evolution (MAVEN) mission has obtained comprehensive particle and magnetic field measurements. The Solar Wind Electron Analyzer (SWEA) provides electron energy-pitch angle distributions along the spacecraft trajectory that can be used to infer magnetic topology. This study presents pitch angle-resolved electron energy shape parameters that can distinguish photoelectrons from solar wind electrons, which we use to deduce the Martian magnetic topology and connectivity to the dayside ionosphere. Magnetic topology in the Mars environment is mapped in three dimensions for the first time. At low altitudes $(<400 \mathrm{~km})$ in sunlight, the northern hemisphere is found to be dominated by closed field lines (both ends intersecting the collisional atmosphere), with more day-night connections through cross-terminator closed field lines than in the south. Although draped field lines with $\sim 100-\mathrm{km}$-amplitude vertical fluctuations that intersect the electron exobase $(\sim 160-220 \mathrm{~km})$ in two locations could appear to be closed at the spacecraft, a more likely explanation is provided by crustal magnetic fields, which naturally have the required geometry. Around $30 \%$ of the time, we observe open field lines from 200-400 km, which implies three distinct topological layers over the northern hemisphere: closed field lines below $200 \mathrm{~km}$, open field lines with footpoints at lower latitudes that pass over the northern hemisphere from $200-400 \mathrm{~km}$, and draped IMF above $400 \mathrm{~km}$. This study also identifies open field lines with one end attached to the dayside ionosphere and the other end connected with the solar wind, providing a path for ion outflow.
\end{abstract}

\title{
1. Introduction
}

One of the most significant findings of the Mars Global Surveyor (MGS) mission was the discovery of strong, localized crustal magnetic fields [Acuna, et al., 1998]. These fields were partially mapped at altitudes ranging from 100 to $180 \mathrm{~km}$ during the 1.4 -year aerobraking period, mostly over the north pole and the sunlit hemisphere. The crustal field was fully sampled during $>7$ years in the $\sim 400-\mathrm{km}$-altitude, $2 \mathrm{am} / 2 \mathrm{pm}$ circular mapping orbit (Figure 1, from Connerney et al. [2005]). These localized crustal fields strongly influence the interaction between solar wind and the Martian space environment, resulting in a complicated and dynamic magnetic topology [e.g., Brain et al., 2003; Harnett and Winglee, 2005; Liemohn et al., 2007; Ma et al., 2014a; Fang et al., 2015].

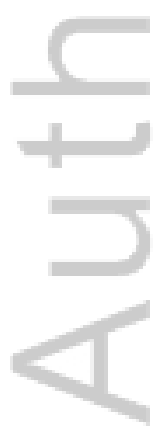




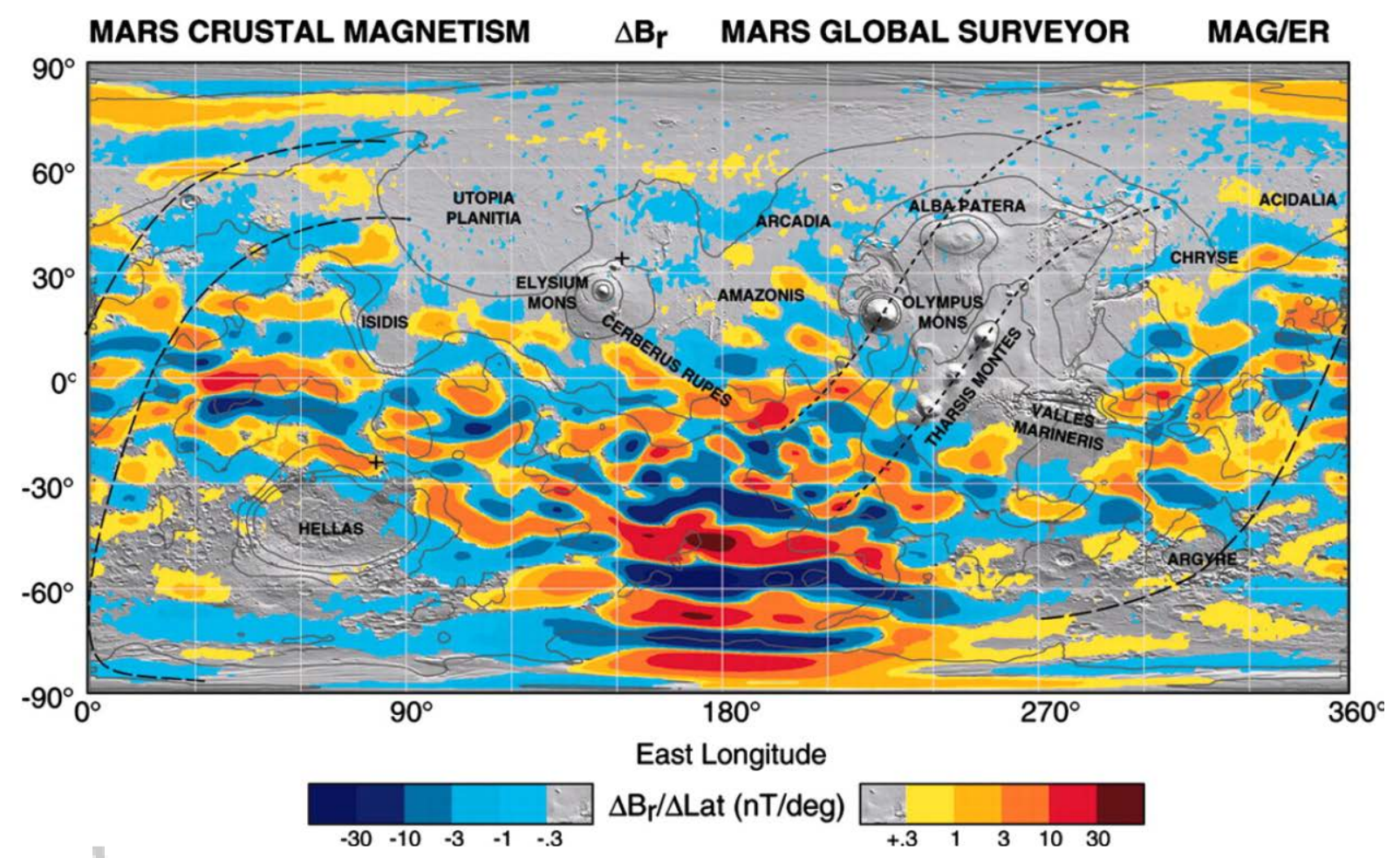

\section{J. E. P. Connemey et al. PNAS 2005; 102:14970-14975}

Figure 1. The map of the derivative of the radial magnetic field along the MGS spacecraft track at a nominal 400-km altitude, also Figure 1 of Connerney et al. [2005]. Copyright (2005) National Academy of Sciences, USA.

Magnetic topology is essential for understanding the Mars plasma environment, which can be categorized into three types: closed, open, and draped field lines. Closed field lines (both ends intersecting the collisional atmosphere) isolate ionospheric plasma from solar wind plasma and allow transport of ionospheric photoelectrons from one location to another. Open field lines, with one end intersecting the collisional atmosphere and the other end connected to the solar wind, permit particle/energy exchange between the Martian ionosphere and the solar wind. Energetic electron precipitation [e.g. Lillis and Brain, 2013; Xu et al., 2015a; Shane et al., 2016] through open fields can cause ionization [e.g. Lillis et al., 2009; Fillingim et al., 2007; Fillingim et al., 2010], heating [e.g., Krymskii et al., 2002, 2004], and excitation (probably aurora [e.g. Bertaux et al., 2005; Brain et al., 2006; Liemohn et al., 2007; Leblanc et al., 2008, Shane et al., 2016]). Open magnetic field lines attached to the dayside ionosphere also provide possible passages for ion escape [e.g. Lillis et al., 2015]. For example, cold ions may be accelerated by the ambipolar electric fields to reach the escape velocity [e.g. Collinson et al., 2015], resembling 
the polar wind at Earth [e.g., Ganguli 1996; Khazanov et al., 1997; Glocer et al., 2009]. Harada et al. [2016] investigated narrowband whistler mode waves in the Martian magnetosphere observed by MAVEN, which were generated by cyclotron resonance with anisotropic electrons on open or closed field lines. These waves in return could also cause electron scattering and precipitation. Draped field lines (both ends connected to the solar wind) can dip low enough into the atmosphere to allow energy transfer through collisions [e.g., Liemohn et al., 2006a].

At Mars, superthermal electrons, mainly consisting of ionospheric photoelectrons and solar wind electrons, are typically magnetized (with the gyrocenters of their helical motion constrained to follow magnetic field lines) and are therefore useful for deducing magnetic topology. Brain et al. [2007] used electron pitch angle distributions measured by the Magnetometer/Electron Reflectometer (MAG/ER) [Acuna et al., 1992; Mitchell et al., 2001] to determine if a magnetic field is closed, open, or draped. The presence or absence of loss cones, which indicate field line intersection with the collisional atmosphere, were used to infer topology. For example, a one-sided loss cone indicates an open field line; a double-sided loss cone, an isotropic photoelectron spectrum, and a superthermal electron void (extremely low count rate) on the nightside [e.g. Mitchell et al., 2001; Steckiewicz et al., 2015; Shane et al., 2016], are all indicators of closed field lines, and a solar wind spectrum with no loss cones indicates a draped field line. Based on this technique, Brain et al. [2007] found that, at $400 \mathrm{~km}$, the dominant field topology was draped/open in the northern hemisphere and closed over the southern strong crustal field regions with cusps in between, where the field has a large radial component. Additionally, the size of loss cone can be used to derive the crustal field strength at the absorption altitude $(\sim 160 \mathrm{~km})$ of these energetic electrons [Lillis et al., 2004; Liemohn et al., 2006; Mitchell et al., 2007], also known as the superthermal electron exobase [e.g. Xu et al., 2016a].

Another way to infer magnetic topology is to use superthermal electron energy distributions to identify the source(s) of electrons traveling parallel and antiparallel to the field line. For example, Liemohn et al. [2006a] and Frahm et al. [2006] reported ionospheric photoelectrons in the high-altitude Martian tail, observed by the Analyzer of Space Plasma and Energetic Atoms (ASPERA-3) experiment [Barabash et al., 2006] onboard the Mars Express spacecraft. Liemohn et al. [2006b] suggested that these observed high-altitude photoelectrons escape down the tail through open field lines with one end embedded in the dayside ionosphere. Frahm et al. [2010] mapped these tail photoelectrons and estimated the escape rate. On the other hand, narrow spikes of electrons fluxes have been observed by both MGS [e.g. Mitchell et al., 2001] and Mars Express [e.g. Dubinin et al., 2008] over the strong crustal regions, which are thought to be solar wind electron precipitation along open field lines. Several studies have 
statistically investigated the dependence of this precipitation on external conditions as well as the effects on the atmospheric target [e.g., Brain et al., 2005; Lillis and Brain, 2013; Xu et al., 2015a; Shane et al., 2016].

Electrons can only be used to infer topology where their motion is governed by electric and magnetic fields. We define a "footpoint" as the location where a magnetic field line intersects the superthermal electron exobase. Below the footpoint, electron motion is dominated by collisions rather than by the magnetic field, so that we can no longer deduce topology from energy-pitch angle distributions. As a specific example, we cannot distinguish between a weak crustal magnetic field line that extends above the electron exobase and a draped solar wind field line that dips below the electron exobase at low solar zenith angles. Additional information, such as the strength and orientation of the magnetic field at the spacecraft, is needed to infer the most likely scenario. In this study, we define magnetic topology based on whether a locally measured flux tube intersects the electron exobase.

Previous missions have greatly improved our understanding of Martian magnetic topology; however, because of limitations in orbit geometry and science instrumentation, there has been no systematic mapping of magnetic topology at altitudes below $300 \mathrm{~km}$ until the Mars Atmosphere and Volatile Evolution (MAVEN) mission [Jakosky et al., 2015]. MAVEN carries a comprehensive set of plasma and field instruments and has a periapsis as low as $\sim 150 \mathrm{~km}(\sim 120$ km during "deep dips"), which is below the superthermal electron exobase. Xu et al. [2016b] reported ionospheric photoelectrons observed in the deep nightside (SZA>120 $)$ below $150 \mathrm{~km}$ by SWEA [Mitchell et al., 2016] onboard MAVEN, which indicated the existence of closed magnetic field lines that straddle the terminator in the northern hemisphere, allowing photoelectron transport from day to night. In this study, we analyze all electron energy/pitch angle distributions obtained to date by SWEA to statistically investigate Mars' magnetic topology down to the superthermal electron exobase over wide ranges of solar zenith angle, local time, longitude and latitude. The instrumentation is described in Section 2. Then, Sections 3 and 4 present how to use electron data, from which the pitch angle-resolved shape parameters are obtained, to deduce the magnetic topology, followed by the maps of different field line types in Section 5. Sections 6 and 7 are discussion and conclusions, respectively.

\section{Instruments}

The MAVEN mission aims to understand the loss of the Mars' atmosphere to space at the current epoch and over the planet's history. MAVEN has an elliptical orbit with an apoapsis of 2.8 Mars radii $\left(\mathrm{R}_{\mathrm{M}}\right)$ and a periapsis of $\sim 150 \mathrm{~km}$ altitude, with several week-long "deep dips", 
which sample key latitudes and local times down to $\sim 120-\mathrm{km}$ altitude. The inclination of the orbit is 74 degrees, and the orbit period is 4.5 hours.

SWEA is a symmetric hemispheric electrostatic analyzer with deflectors that measures the energy/angle distributions of electrons from 3 to $2000 \mathrm{eV}$ over $\sim 80 \%$ of the sky, and electrons from 2000 to $4600 \mathrm{eV}$ with a field of view that shrinks with energy. The 64 logarithmically spaced energy bins provide $12 \%(\Delta \mathrm{E} / \mathrm{E})$ sampling over the full range, which slightly oversamples the instrumental energy resolution of $17 \%$. This is sufficient to distinguish ionospheric photoelectrons from (possibly energized) electrons of solar wind origin. The field view is divided into 96 solid angle bins, providing $\sim 20^{\circ}$ resolution. Pitch angle distributions can be obtained from the full energy/angle (3D) distributions, but these have a low cadence (> $16 \mathrm{sec}$ ) because of telemetry rate limitations. The data described here are 2D cuts (great circles) through the 3D distributions that are calculated onboard using real-time MAG data and designed to provide maximum pitch angle coverage, even as the magnetic field direction varies. This PAD (pitch angle distribution) data product is 6 times smaller than the 3D product and is provided with a 2-4 sec cadence, depending on altitude. See Mitchell et al. [2016] for a more detailed description.

The Magnetometer (MAG) is comprised of two independent tri-axial fluxgate sensors located on extensions ("diving boards") at the ends of the solar panels. Each magnetometer measures the vector field with an accuracy of $\sim 0.1 \mathrm{nT}$ (including corrections for dynamic fields generated on the spacecraft) at a cadence of 1/32 sec. More details about the MAG instrument are provided by Connerney et al. [2015].

\section{Superthermal Electrons and Magnetic Topology}

The interaction of solar wind with the Martian ionosphere and crustal anomalies gives rise to several types of magnetic topology. The theoretical predictions of such a complex interaction from a time-dependent multispecies Mars-magnetohydrodynamics (MHD) simulation [Ma et al., 2014b; Fang et al., 2015] is shown in Figure 2. The multispecies single-fluid MHD [Ma et al., 2004] includes four continuity equations for four ion species, $\mathrm{H}^{+}, \mathrm{O}_{2}{ }^{+}, \mathrm{O}^{+}, \mathrm{CO}_{2}{ }^{+}$, but assumes all the ions share the same velocity and temperature. Details of the model are described in Ma et al. [2004]. This particular time-dependent run let the planet rotate for 26 hours under quiet solar wind conditions, with a solar wind density of $4 \mathrm{~cm}^{-3}$, a velocity of $400 \mathrm{~km} / \mathrm{s}$, a Parker spiral IMF of $-3 \mathrm{nT}$ and $56^{\circ}$ in the MSO (Mars-centered Solar Orbital) X-Y direction, and a plasma temperature of $3.5 \times 10^{5} \mathrm{~K}$. The simulation was performed in the MSO coordinates, the $\mathrm{X}$ axis points from the center of Mars to the Sun, the $\mathrm{Y}$ axis is opposite to the orbital motion of 
Mars, and the $\mathrm{Z}$ axis is perpendicular to the Mars' orbital plane. Detailed setup of the simulation is described in Ma et al. [2014b].

Figure 2 shows an example of the field line tracing starting at $150 \mathrm{~km}$ in altitude at a specific time point when the strong crustal fields are on the dayside from two perspectives (left view from the Sun and right towards the Sun). The color contour on the spherical surface shows the magnetic magnitude at $150 \mathrm{~km}$ altitude. Different types of field lines are highlighted with colors: purple for closed field lines with both footpoints on the dayside, black for closed field lines with both footpoints on the nightside, green for one footpoint on the dayside (solar zenith angle, $\left.\mathrm{SZA}<90^{\circ}\right)$ and the other on the nightside $\left(\mathrm{SZA}>90^{\circ}\right)$, orange for open field lines attached to the dayside ionosphere, blue for open field lines attached to the nightside. Draped field lines are not present in this case because the tracing starts at $150 \mathrm{~km}$ and, according to our definition above, we are treating all field lines crossing this altitude (i.e., the electron exobase) as either open or closed. Each type of field lines has access to different electron populations, photoelectrons or solar wind electrons, in each end. This information of electron populations in return can be utilized to retrieve the magnetic topology.

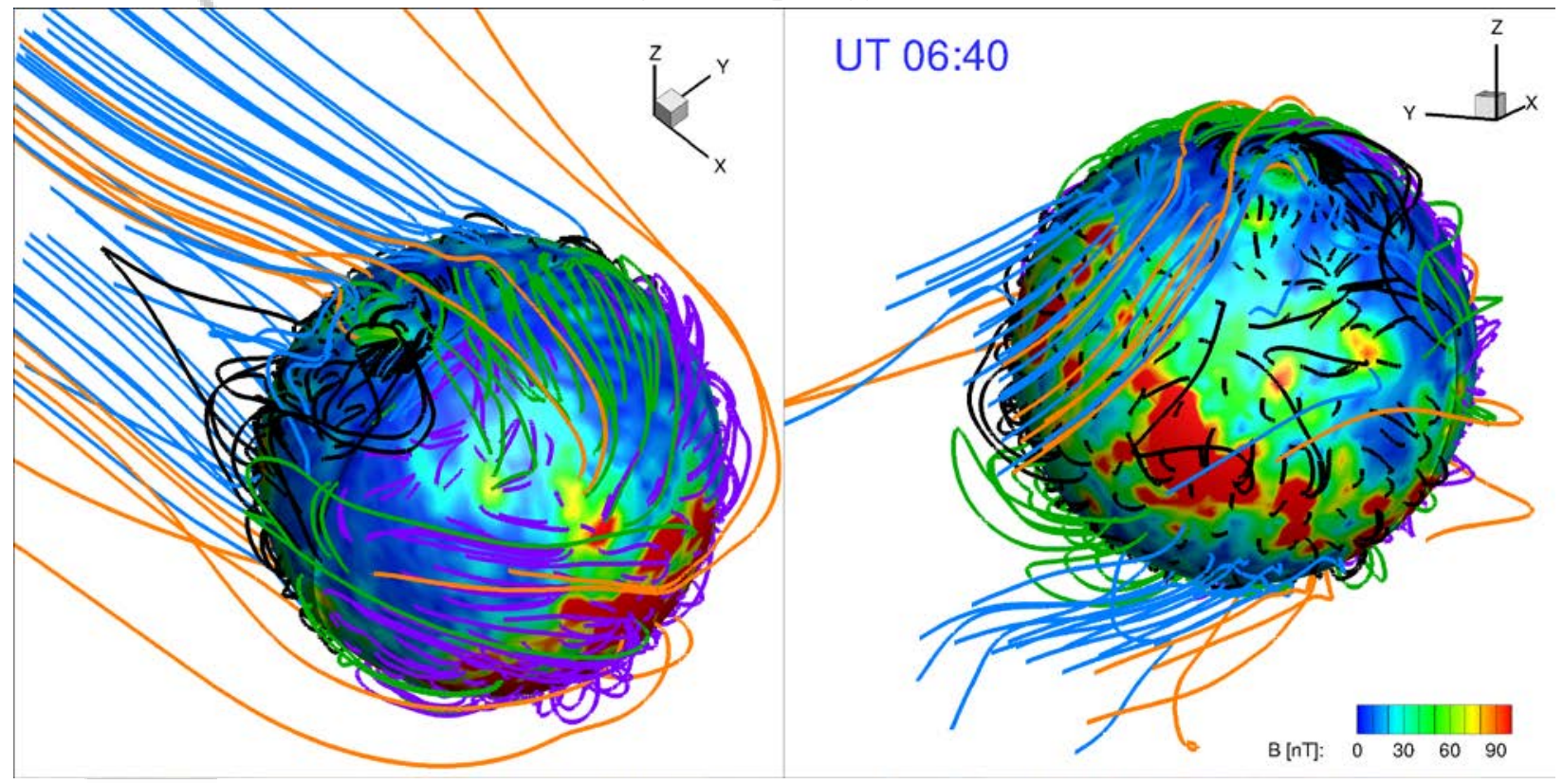

Figure 2. Field line tracing at UT 06:40 (the simulation starts at UT 00:00) from two perspectives (left and right), with the strong crustal fields on the dayside. The color on the spherical surface is the magnetic magnitude at $150 \mathrm{~km}$. Different types of field lines are highlighted with colors, described in details in the text. The field lines are extracted from the simulation result in Ma et al. [2014b]. 
As mentioned above, SWEA has a fine energy resolution to distinguish ionospheric photoelectrons from solar wind/magnetosheath electrons based on their energy spectral shape. As noted in several studies [e.g. Mitchell et al., 2000; Liemohn et al., 2003; Frahm et al., 2006], the Martian photoelectron energy spectrum has a several distinct features, corresponding to features in solar irradiance [e.g. Xu et al., 2015b; Peterson et al., 2016]: (1) a cluster of sharp peaks from 22 to $27 \mathrm{eV}$ due to ionization of $\mathrm{CO}_{2}$ and $\mathrm{O}$ by the intense He II 30.4-nm ( 40 eV) solar line, (2) a sharp drop in flux from 60 to $70 \mathrm{eV}$ (the photoelectron knee) due to a corresponding sharp decrease of solar irradiance at wavelengths shorter than $17 \mathrm{~nm}$, (3) a peak near $500 \mathrm{eV}$ produced by ionization of oxygen $\mathrm{K}$-shell electrons by soft $\mathrm{X}$ rays (and subsequent relaxation of the resulting excited ion by the emission of photons and Auger electrons), and (4) a second sharp decrease in electron flux at energies just above the Auger peak due to another drop in solar irradiance. In contrast, these features are absent in the energy spectra of solar wind electrons in all regions of the Mars' plasma environment. Although SWEA cannot resolve the cluster of photoelectron peaks from 22 to $27 \mathrm{eV}$, the energy resolution is sufficient to readily distinguish between photoelectrons and solar wind electrons.

To infer magnetic topology, the basic idea of this study is to examine what electron population, ionospheric photoelectrons vs. solar wind electrons, is measured in the parallel and anti-parallel directions. The topology criteria are slightly different for the dayside and nightside hemispheres. In this study, we define the dayside as solar zenith angle (SZA) $<90^{\circ}$ and the nightside as $\mathrm{SZA}>110^{\circ}$ to ensure that the ionosphere near and below the electron exobase is in darkness [cf., Shane et al, 2016]. On the dayside, a closed field (purple lines in Figure 2) is defined as one on which photoelectrons being measured in both parallel and anti-parallel directions. Closed field lines with both ends intersecting the collisional dayside ionosphere fill with photoelectrons and are simultaneously isolated from solar wind electrons. An open field line (orange lines in Figure 2) is identified as having photoelectrons in one direction and solar wind electrons in the other, as one end of the field is attached to the ionosphere and the other to solar wind; the draped field (not shown in Figure 2) is designated when solar wind/sheath electrons are found in both directions, as the field line connects to the solar wind on both ends.

In the darkness of the nightside ionosphere, there is no photoelectron production, so we use a different set of criteria. There are two types of closed field lines on the nightside: (1) one footpoint on the dayside and another on the nightside (a cross-terminator closed field line, green lines in Figure 2), and (2) both footpoints on the nightside (black lines in Figure 2). In the first case, photoelectrons are produced on the dayside, travel across the terminator along the field line (above the electron exobase), and precipitate into the nightside. Part of the returning flux is magnetically reflected, while the more field aligned flux suffers collisions with the neutral 
atmosphere, forming a loss cone. We denote photoelectrons flowing towards the planet as a closed field line. In the second case, there is no photoelectron production at either footpoint and no access for solar wind electrons. Superthermal electron fluxes in both directions are $\sim 2$ orders of magnitude lower than typical fluxes of either photoelectron or solar wind electron populations. We define this situation as a superthermal electron void.

-For open field lines, one end is connected to the solar wind while the other intersects the electron exobase on either the dayside (orange lines in Figure 2) or the nightside (blue lines in Figure 2). We identify the first case by observing photoelectrons flowing away from the planet and solar wind electrons flowing towards the planet. We identify the second case as measuring solar wind electrons in both directions, which can arise from solar wind electrons traveling towards the planet and magnetically reflected and/or backscattered electrons traveling in the opposite direction. Thus, one drawback of this particular methodology is that we are unable to differentiate the open field lines attached to the nightside from draped solar wind magnetic fields.

On the dayside, we organize our results into three topological categories: closed, open, and draped (Table 1), which correspond with the definitions used by previous authors. However, on the nightside, because we infer topology based on the presence of ionospheric photoelectrons (which are produced in sunlight), we use the following restricted definitions for these categories. "Closed" refers specifically to trans-terminator closed field lines, with one footpoint on the dayside and the other on the nightside. "Open" refers specifically to field lines with one footpoint on the dayside and the other end connected to the IMF. "Draped" refers to field lines that are connected to the IMF on both ends (the normal definition), but also includes open field lines with one footpoint on the nightside and the other connected to the IMF. In the latter case, solar wind electrons are observed in both directions because of backscatter and/or magnetic reflection. "Voids" are a second category of closed field line with both footpoints on the nightside.

\begin{tabular}{|l|l|l|}
\hline & Dayside $\left(\mathrm{SZA}<90^{\circ}\right)$ & Nightside $\left(\mathrm{SZA}>110^{\circ}\right)$ \\
\hline Closed & Ph e- in both directions (purple) & $\begin{array}{l}\text { Ph e- traveling towards the planet } \\
(\text { green })\end{array}$ \\
\hline Open & $\begin{array}{l}\text { Ph e- in one direction; SW e- in the } \\
\text { opposite direction (orange) }\end{array}$ & $\begin{array}{l}\text { Ph e- traveling away from the planet; } \\
\text { SW e- traveling towards the planet } \\
(\text { orange })\end{array}$ \\
\hline Draped & SW e- in both directions (draped IMF) & $\begin{array}{l}\text { SW e- in both directions (blue and } \\
\text { draped IMF) }\end{array}$ \\
\hline Void & N/A & Low omni-directional electron fluxes \\
\hline
\end{tabular}


\begin{tabular}{|l|l|l}
\hline (closed) & (black)
\end{tabular}

Table 1: Criteria for determining magnetic field topology (closed, open, draped, and void) based on electron populations traveling parallel and anti-parallel to the magnetic field on the dayside and the nightside, respectively. Photoelectron is denoted as "Ph e-" and solar wind/sheath electron as "SW e-". The colors in parenthesis indicate the field lines in Figure 2.

\section{Shape Parameter}

To systematically distinguish ionospheric photoelectrons from solar wind electrons, we have designed a shape parameter to identify the He II peaks and the photoelectron knee in the measured energy spectra. We manually selected sixty photoelectron energy spectra and then calculated the derivative of the electron fluxes with respect to energy $\log$ space $(\mathrm{d}(\log \mathrm{F}) / \mathrm{d}(\log \mathrm{E}))$ using the three-point Lagrangian interpolation for each spectrum. This differentiation removes overall changes in the electron flux caused by variations in solar irradiance [e.g. Banks and Nagy, 1970; Xu and Liemohn, 2015] and the neutral atmospheric composition [e.g. Xu et al., 2014; Xu et al., 2015c] and also highlights sharp features in the spectral shape of photoelectrons, such as the He II peaks and the photoelectron knee, which are observed in photoelectrons but not the solar wind. We average the sixty derivatives to produce a template with good counting statistics (Figure S1, black squares). For any measured electron distribution, we can calculate the electron flux derivatives and compare with the template. We define the shape parameter as the sum of the absolute differences between the measured derivative and the template from $20 \mathrm{eV}$ to $80 \mathrm{eV}$. The more similar the observed derivative is to the template, the smaller the shape parameter and the more likely that the observed distribution contains photoelectrons. Figure S1 shows how a photoelectron observation (red) follows the template and has a small shape parameter, while a solar wind observation (blue) fails to capture the two sharp photoelectron features and has a large shape parameter. Although the shape parameter is a continuous quantity, since both populations can be present in various proportions on a given field line, we find that a value of unity provides a useful separation of distributions dominated by photoelectrons (shape parameter $<1$ ) and those dominated solar wind electrons (shape parameter $>1$ ). The shape parameter is calculated separately for the parallel $\left(0^{\circ}-60^{\circ}\right.$ pitch angle) and anti-parallel $\left(120^{\circ}-180^{\circ}\right.$ pitch angle $)$ populations. From the local magnetic field direction, we can determine which population is traveling towards the planet and which population is traveling away.

To demonstrate how the shape parameter works, we have selected two orbit examples, for dayside and nightside observations. For the dayside, Figure 3 shows MAVEN measurements made from 05:17 to 06:15 UT (universal time) on April 17, 2015. The panels from top to bottom 
are: the altitude, solar zenith angle (SZA), magnetic field strength and magnetic field components in MSO coordinates measured by MAG, the normalized 111-140-eV electron pitch angle distribution, and the electron energy spectra measured by SWEA (energy fluxes in units of $\mathrm{eV} \mathrm{cm}^{-2} \mathrm{~s}^{-1} \mathrm{sr}^{-1} \mathrm{eV}^{-1}$ ), and shape parameters for electrons moving towards (red) and away from (green) the planet, respectively. The direction of the electrons relative to the planet is determined based on the local magnetic field measurement. During this time range, the spacecraft moves from high altitudes, where the magnetic field is weak, through the periapsis, which is dominated by the crustal fields ( 05:40-05:56 UT), and then back to high altitudes. The three bottom panels simultaneously exhibit systematic, correlated changes. From 05:17 to 05:24 UT, the pitch angle distribution (PAD) is nearly isotropic, while the shape parameters for both directions are above 1. The left panel of Figure 4 shows the parallel and anti-parallel electron spectra obtained at T1, marked by the first dotted vertical line in Figure 3. The electron spectra in both directions are typical solar wind/sheath electron spectra, with no evidence for photoelectron features. The local minimum at $\sim 8 \mathrm{eV}$ is caused by the spacecraft potential (vertical dashed line), which separates spacecraft photoelectrons at lower energies from ambient electrons at higher energies. From 05:30 to 06:00, the electron energy spectrogram (second panel from the bottom) shows one local maximum from 20 to $30 \mathrm{eV}$, corresponding to the He II feature of the photoelectrons, and another one near $500 \mathrm{eV}$, indicative of Auger electrons. Meanwhile, the shape parameters for both directions are below 1 (except for a brief interval from 05:46 to 05:48 UT, which will be described below). An example of the electron spectra in this region is shown in the right panel of Figure 4. Several photoelectron spectral features are present in both directions, including the $\mathrm{He}$ II peaks, the photoelectron knee, and the sharp drop in electron flux above $\sim 500 \mathrm{eV}$. There are also time periods during which the shape parameter is $<1$ for electrons traveling away from the planet and $>1$ for electrons traveling towards the planet, including 05:24-05:30 UT, 05:46-05:48 UT, and 06:02-06:10 UT. During these time intervals, the pitch angle distribution exhibits a onesided loss cone, which is classified as an indicator for open field lines (see Brain et al. [2007]). The parallel and anti-parallel electron spectra for this case are shown in the middle panel of Figure 4. The local magnetic field has an elevation angle (relative to the horizontal plane) of $63^{\circ}$, pointing away from the planet, therefore parallel electrons are flowing away from the planet and anti-parallel electrons towards the planet. This conversion from pitch angles to the direction relative to the planet is implied below based on the local magnetic elevation angle. The spectrum for electrons traveling away from the planet $\left(0^{\circ}-60^{\circ}\right.$ pitch angle, red) shows typical photoelectron features, and the spectrum for electrons traveling in the opposite direction $\left(120^{\circ}\right.$ $180^{\circ}$ pitch angle, blue) is typical for the solar wind. Thus, the pitch angle-resolved shape 
parameter provides a reliable method for determining the source regions of the parallel and antiparallel electron populations, which we use to infer magnetic topology.

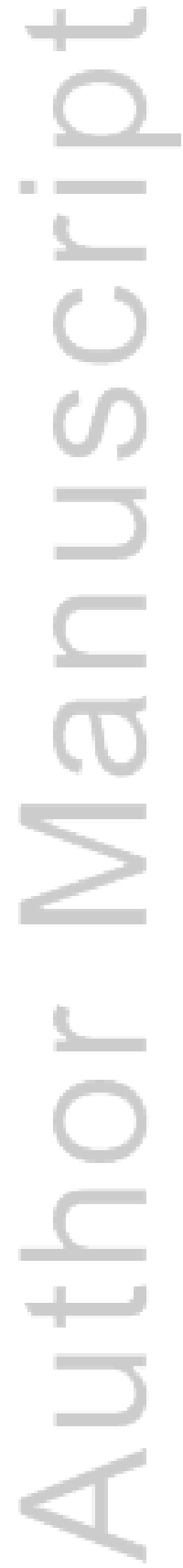


Confidential manuscript submitted to Journal of Geophysical Res., Space Physics
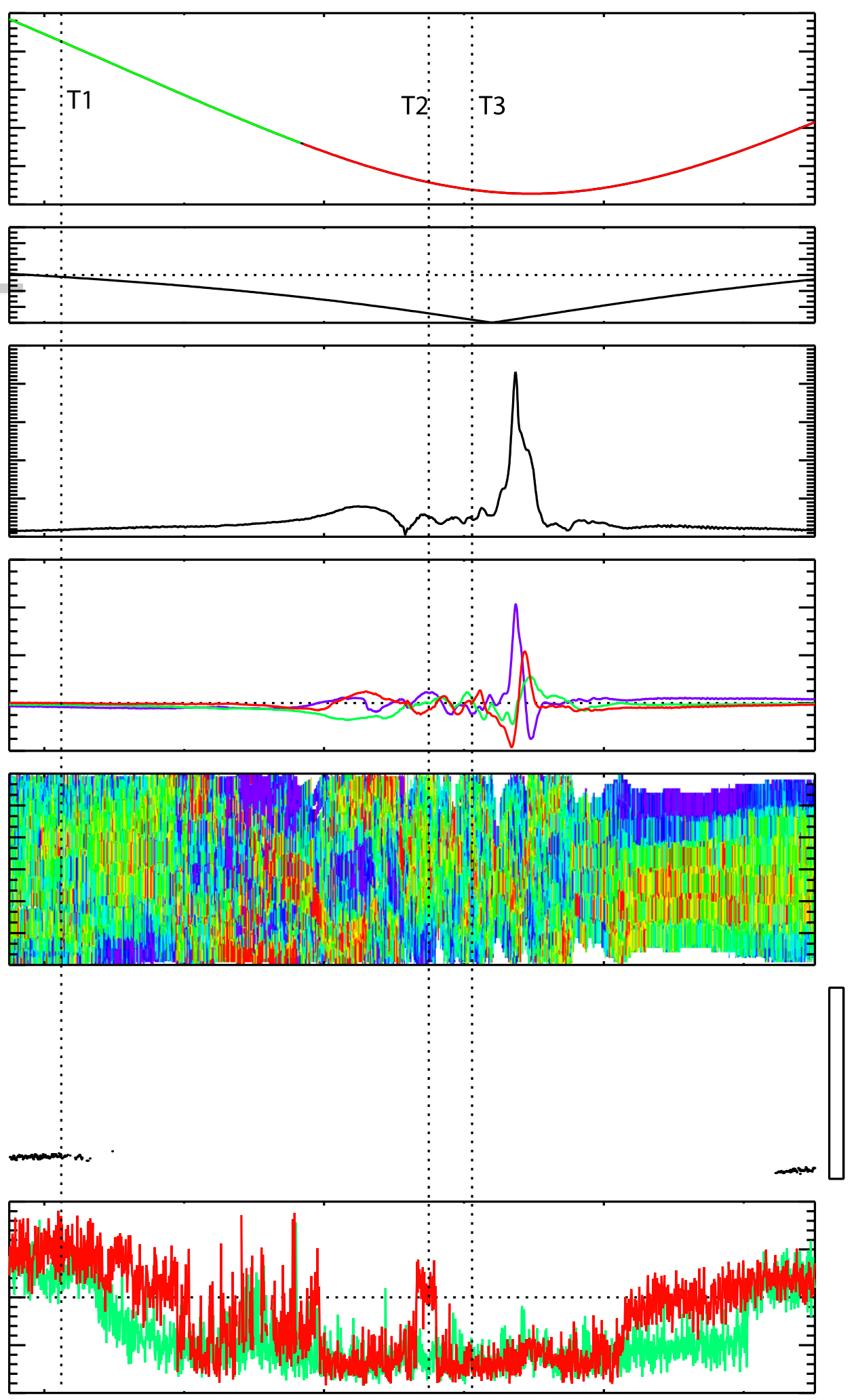

This article is protected by copyright. All rights reserved. 
Figure 3. Time series of the spacecraft altitude, SZA, magnetic field strength, magnetic field components in the MSO coordinates, the normalized pitch angle distribution of 111$140 \mathrm{eV}$ electrons, the energy spectra, and shape parameters for electrons moving towards (red) and away from (green) the planet, from top to bottom, respectively. The blue, green and red colors in the altitude panel highlight the theoretical region for the optical shadow, magnetosheath and the pileup region based on fittings of the bow shock and the magnetic pileup boundary [Trotignon et al., 2006]. Three dashed vertical lines mark the time of extracted electron energy spectra in Figure 4. The black dots in the electron energy spectrogram panel indicate the spacecraft potential estimated by SWEA.
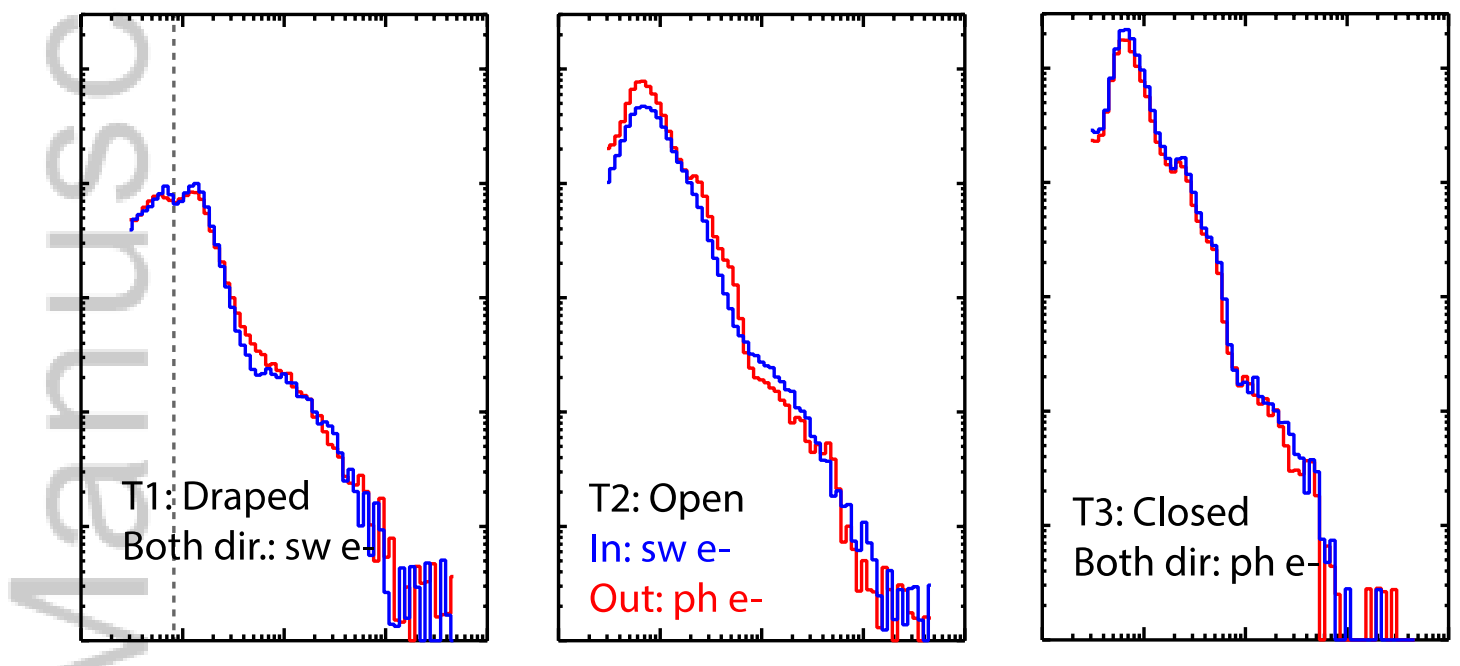

Figure 4. Electron energy spectra for the parallel (red) and anti-parallel (blue) directions measured by SWEA. The red spectrum is averaged over pitch angles $0-60^{\circ}$ and the blue spectrum over pitch angles $120^{\circ}-180^{\circ}$. The vertical dashed line in the left panel marks the spacecraft potential. The altitude and SZA of the measurement, as well as the azimuthal (in the horizontal plane) and elevation angles (relative to the horizontal plane) of the local magnetic field, are shown in the upper right corner. The three panels corresponding to the time marked out by the three dotted vertical lines in Figure 3.

For the nightside, an example orbit on Feb. 6, 2015 is shown in Figure 5, in the same format as Figure 3. The spacecraft was on the nightside (SZA>110 $)$ from 13:47 to 14:28 UT. Three parallel/anti-parallel spectral pairs, selected at times marked by the dotted vertical lines in Figure 5, are chosen and shown in Figure 6. For T1, both shape parameters are $<1$ (Figure 5) and 
in the left panel of Figure 6. Electrons traveling towards the planet exhibit apparent photoelectron spectral features (Figure 6, left panel, red spectrum), interpreted as photoelectrons precipitating into the nightside on a closed field line that straddles the terminator. The spectrum of electrons traveling in the opposite direction (blue) show only faint He II peaks and no clear evidence for a photoelectron knee. This pair of spectra indicate a closed field line with one footpoint in the dayside ionosphere and the other footpoint in darkness. Photoelectrons produced at the sunlit footpoint travel along the field line and precipitate onto the dark footpoint. A fraction of the precipitating flux is backscattered, with the photoelectron features washed out mainly by inelastic collisions. This measurement, however, was made near the terminator, and a better example for such a scenario is shown in Xu et al. [2016b]. More often than not, the spectrum of backscattered photoelectrons is too washed out to be identified by the shape parameter. Thus, for the nightside, the criterion for a closed field line is only that precipitating electrons have a shape parameter less than 1 . For T2, the shape parameters are all $>1$ and the spectra for both directions (Figure 6, the middle panel) are solar wind/sheath-like. There are several time intervals when the shape parameter for electrons traveling away from the planet dips below 1 and remains above 1 for electrons traveling towards the planet. One example marked as T3 is shown in the right panel of Figure 6. The outflowing flux has a photoelectron spectrum (blue), while precipitating flux has a solar wind/sheath-like spectrum (red). To have access both populations, the field line has to have a footpoint on the dayside, is pulled back to the nightside (where the measurement is made), and opens to the solar wind (see the yellow lines in Figure 2). Such open field lines have access to the dayside ionosphere and provide a path for ion escape. The last type of topology, superthermal electron voids, is another example of closed field lines, which exists mostly on the nightside. This can be seen in Figure 5, during the time intervals 13:52-13:53 UT, and 13:54-13:56 UT, corresponding to extremely low electron fluxes (at or close to the background level at most energies) as well as the absence of shape parameters in both directions. (Our software tags shape parameters for such intervals as undefined.) A reliable method of identifying these regions is to set an energy flux threshold of $10^{5} \mathrm{eV} \mathrm{cm}^{-2} \mathrm{~s}^{-1} \mathrm{sr}^{-1} \mathrm{eV}^{-1}$ at an energy of $40 \mathrm{eV}$. Observed fluxes below this threshold are identified as voids.

The two example orbits have demonstrated that the pitch-angle resolved shape parameter is reliable to infer the magnetic topology. However, a complication is that the shape parameter is a gradually increasing, instead of binary, number to represent changing from photoelectrons to solar wind electrons. The threshold of 1 used in this study is reasonable but we have tested other thresholds, $0.7,0.8,1.2$, and 1.4. The overall findings of this study stay the same but the occurrence rate for each type of topology changes with different thresholds, as expected. In particular, when shape parameter is close to 1 , it might be a mixed spectrum of both 
photoelectrons and solar wind/sheath electrons, for example having both the He II feature and a less prominent flux drop near the photoelectron knee, or a degraded spectrum like the blue line in the left panel in Figure 6. In addition, when the magnetic elevation angle is small, a small perturbation in the magnetic field can change the field line direction, then the classification of away/towards for the shape parameter, consequently the determination of the topology. This is also why we do not distinguish solar wind electron flowing towards or away from the planet for open field lines on the dayside (see Table 1). These complications are important to take into consideration to analyze case studies. For this statistical study, the simple classifications in Table 1 are sufficient to obtain magnetic topology maps below.

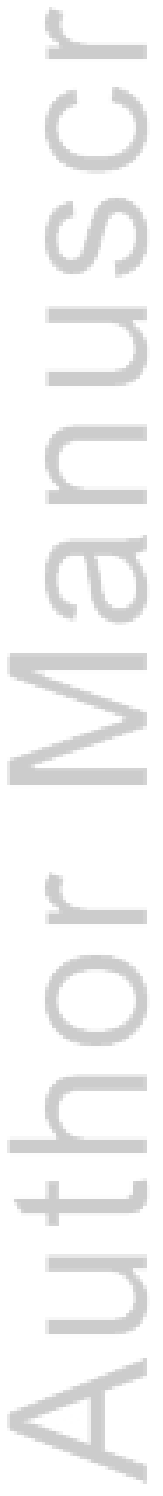



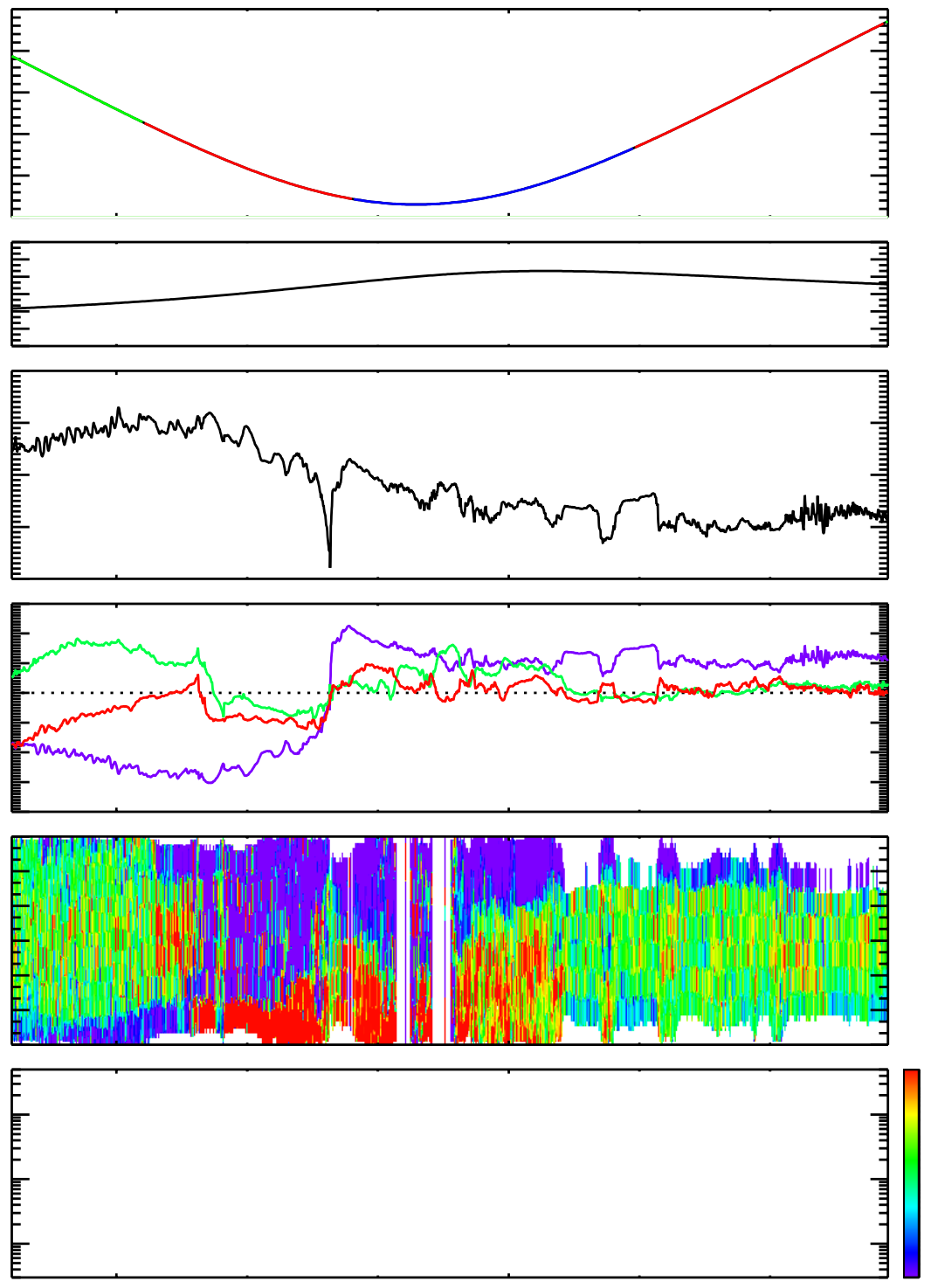

Figure 5. Time series of the spacecraft altitude, SZA, magnetic field strength, magnetic field components in the MSO coordinates, the normalized pitch angle distribution of 111-

This article is protected by copyright. All rights reserved. 
$140 \mathrm{eV}$ measured, the energy spectra, and shape parameters for electrons moving towards (red) and away from (green) the planet, from top to bottom, respectively. Same format as Figure 3.
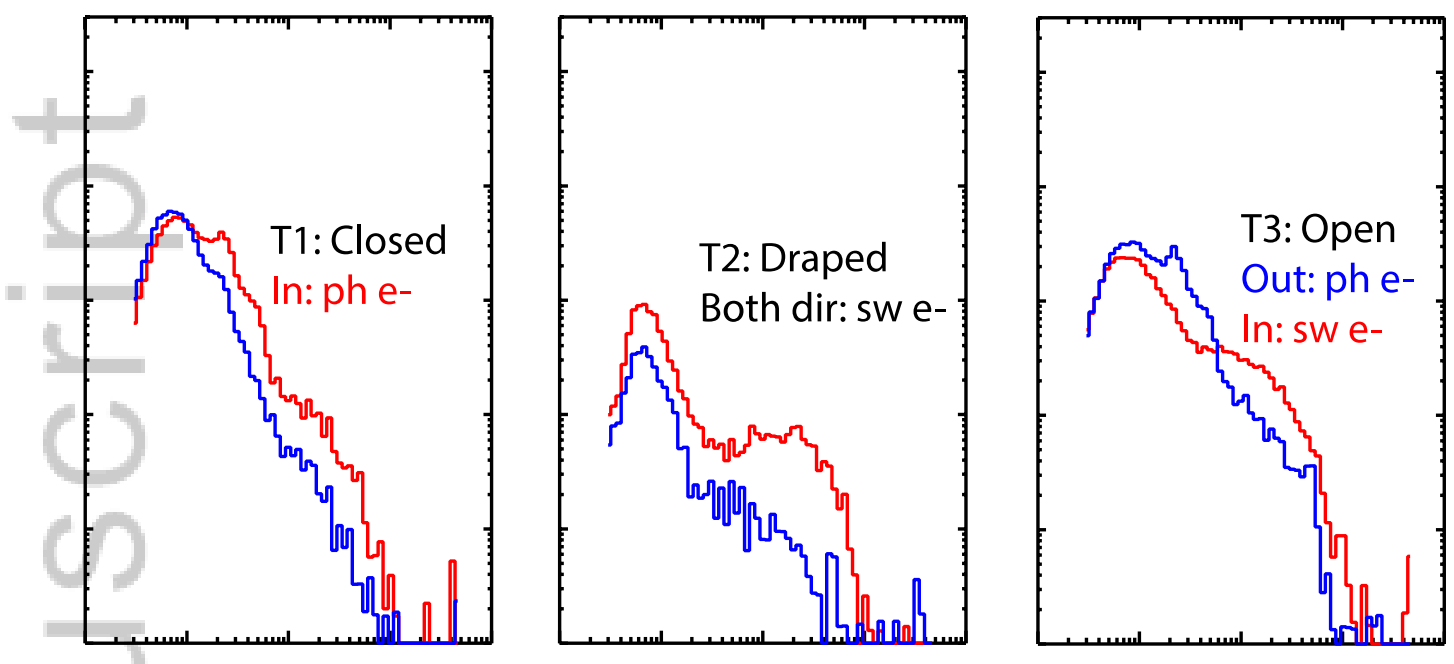

Figure 6. Electron energy spectra for parallel (red) and anti-parallel (blue) directions measured by SWEA. The red spectrum is averaged over pitch angle $0-60^{\circ}$ and the blue spectrum over pitch angle $120^{\circ}-180^{\circ}$. The three panels corresponding to the time marked out by the three dotted vertical lines in Figure 5.

\section{Maps for Magnetic Topology}

Now that we have established the methodology to infer magnetic topology from the pitch angle-resolved shape parameters to determine the magnetic topology, the three-dimensional maps can be created by examining all the available MAVEN data, from Dec. 1, 2014 to May 2, 2016. This study is limited to an altitude range of $160-1000 \mathrm{~km}$ to investigate the crustal field control of the Martian magnetic topology. Above $1000 \mathrm{~km}$ altitude, the strongest crustal magnetic fields are comparable in strength to the solar wind magnetic field [e.g. Brain et al. 2003]. Below $160 \mathrm{~km}$ altitude, collisions become important for superthermal electrons [Xu et al., 2016a], and the pitch angle distribution becomes isotropic, so that our method for inferring topology is no longer valid.

The data are divided into six altitude ranges: $800-1000 \mathrm{~km}, 600-800 \mathrm{~km}, 400-600 \mathrm{~km}$, $300-400 \mathrm{~km}, 200-300 \mathrm{~km}$, and 160-200 km. For each altitude range, the data are further divided into $18^{\circ} \times 10^{\circ}$ geographic longitude-latitude bins. Finally, we divide the data into dayside (SZA < $90^{\circ}$ ) and nightside $\left(\mathrm{SZA}>110^{\circ}\right)$. Although we present data mapped into geographic longitude 
and latitude, it is important to note that each bin contains all local times that fall within the SZA range. The total sample number for each bin (Figure 7) is the sum of all cases identified according to Table 1. Because $20 \%$ of the sky is outside SWEA's field of view, there is occasionally insufficient pitch angle coverage to calculate the parallel or anti-parallel shape parameter. For this reason, $4 \%$ of data are excluded on the dayside and $18 \%$ on the nightside. The percentage of excluded spectra is higher on the night side because the magnetic field direction tends to be close to the Mars-Sun line, and thus near the edge of SWEA's field of view.

Before calculating the shape parameter, it is necessary to correct the electron data for energy shifts caused by the spacecraft potential $\left(\phi_{\mathrm{sc}}\right)$ shifting the electron energy spectra [Mitchell et al., 2016]. When $\left|\phi_{\mathrm{sc}}\right|>4 \mathrm{~V}$, the shift is large enough that the shape parameter for an uncorrected photoelectron spectrum can exceed 1. Depending on spacecraft orientation and plasma environment, the spacecraft potential is typically in the range of -20 to $+10 \mathrm{~V}$. We have corrected the data for spacecraft potential obtained using the methodology described in Mitchell et al. [this issue], which can estimate negative potentials from -16 to $-1 \mathrm{~V}$ in the ionosphere and positive potentials greater than $+3 \mathrm{~V}$ throughout the Mars environment. There is no need to correct for potentials from -1 to +3 Volts; however, some data must be excluded when the potential is more negative than -16 Volts.

Our current understanding of Mars' magnetic environment (based mostly on MGS observations) is that the southern hemisphere is dominated by crustal fields (to an altitude that depends on crustal field strength), while the north is dominated by draped solar wind magnetic fields [Brain et al. 2003, 2006]. For the first time, the MAVEN orbit allows measurements of magnetic topology over wide ranges of local time, longitude, latitude, and altitude (Fig. 7). The altitude ranges from the electron exobase $(\sim 160 \mathrm{~km})$ to $400 \mathrm{~km}$ is of particular interest, because this region was sparsely mapped by MGS, with most of the measurements in the sunlit northern hemisphere. 

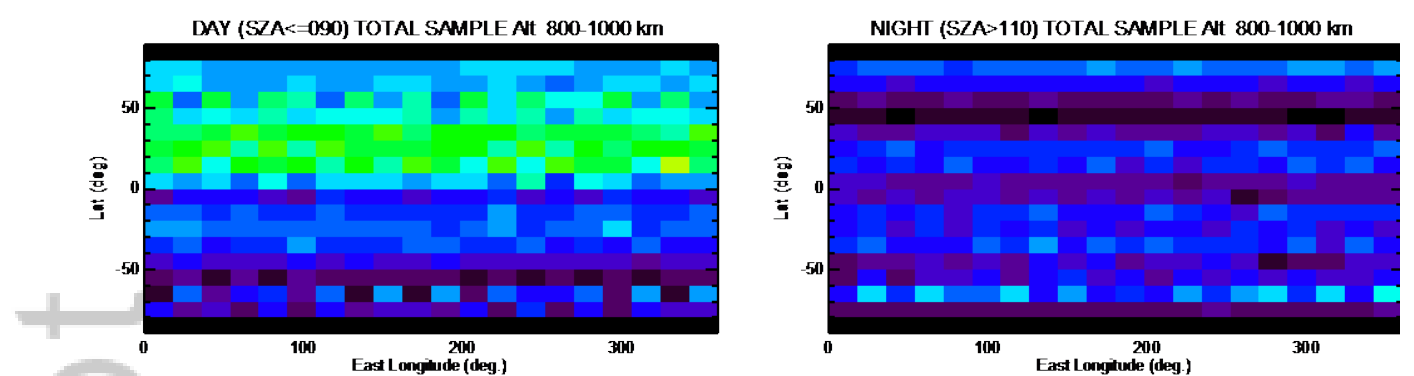

DAY (SZA $=090$ ) TOTAL SAMPLEAK $600-800 \mathrm{~km}$
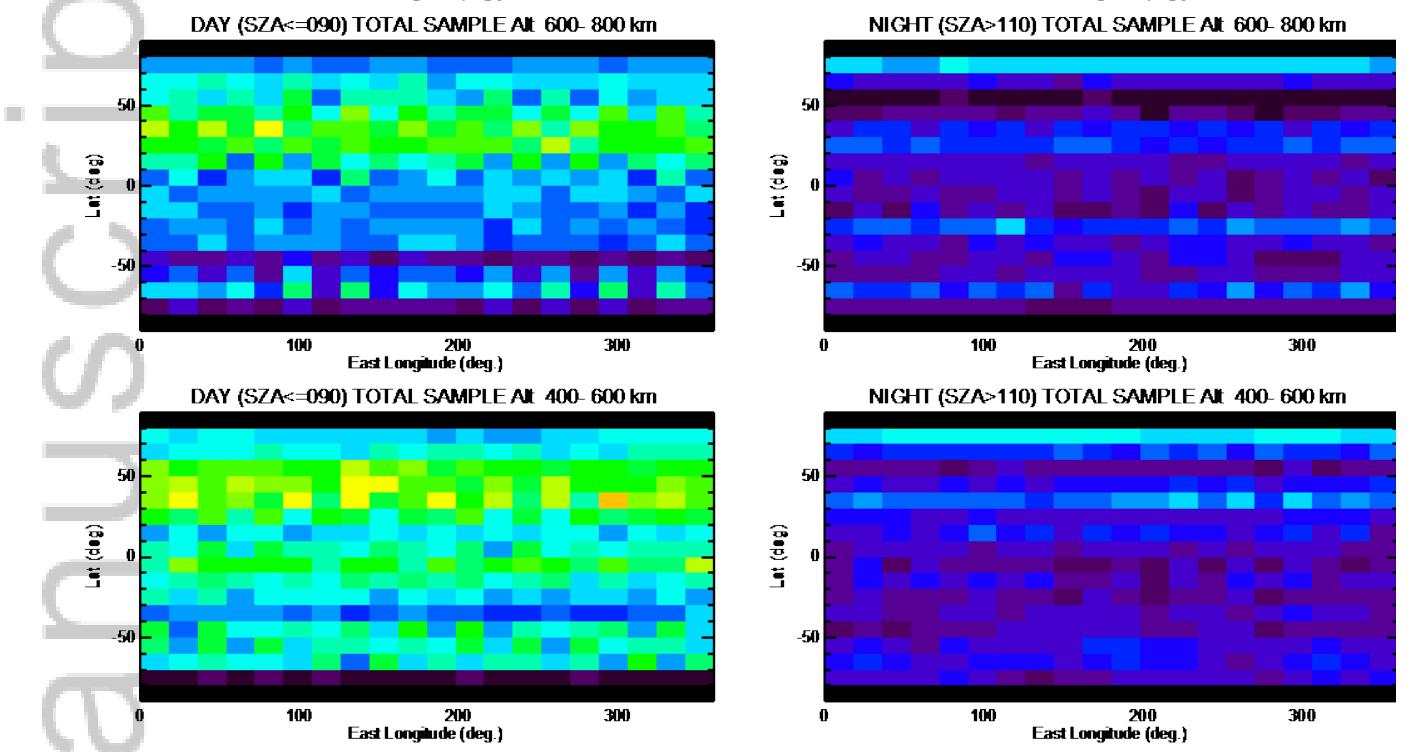

DAY (SZA $=090)$ TOTAL SAMPLEA $300-400 \mathrm{~km}$
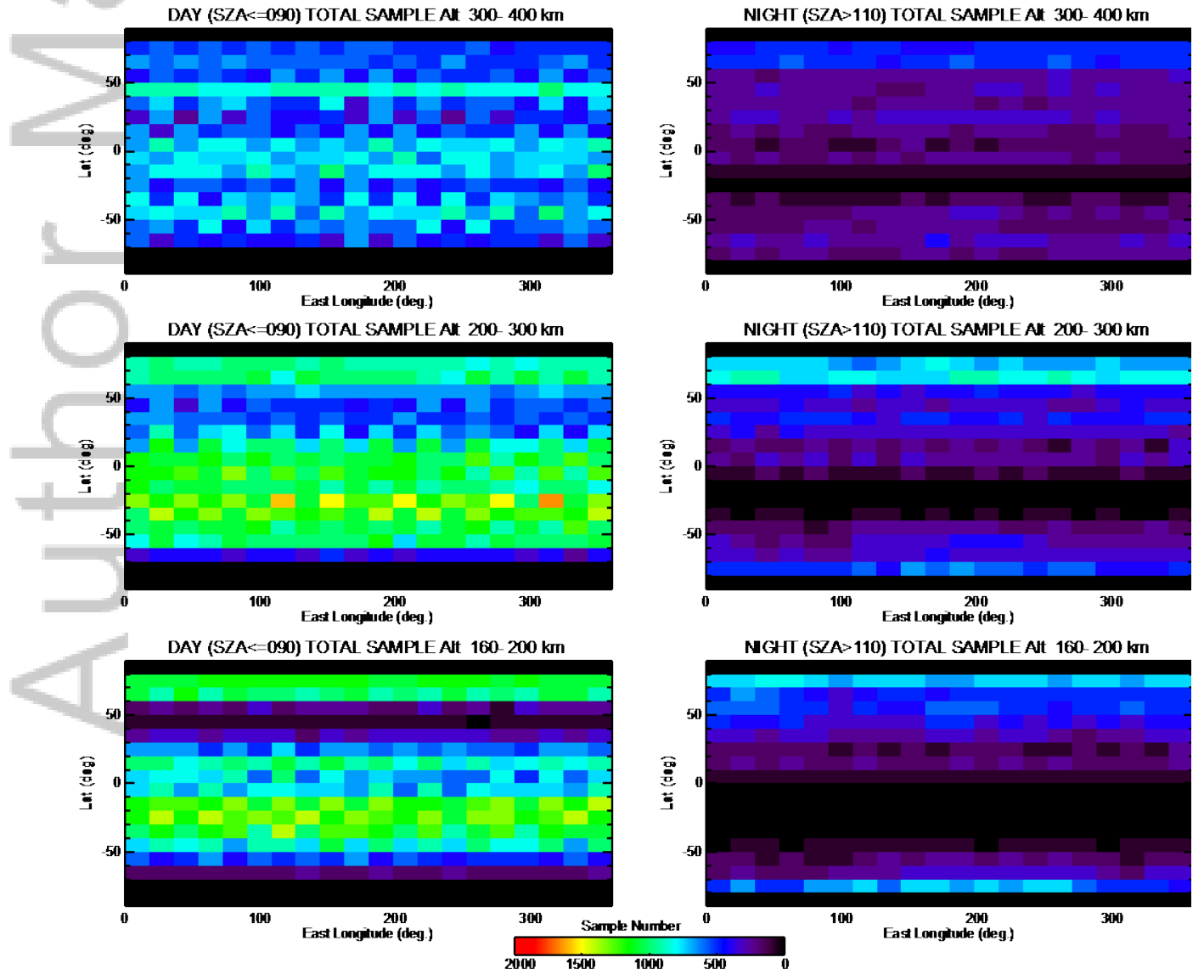

This article is protected by copyright. All rights reserved. 
Figure 7. The total sample number against latitude and longitude. The left column is for dayside $\left(\mathrm{SZA}<90^{\circ}\right)$ and right for nightside $\left(\mathrm{SZA}>110^{\circ}\right)$. From top to bottom, each row is for the altitude range of $800-1000 \mathrm{~km}, 600-800 \mathrm{~km}, 400-600 \mathrm{~km}, 300-400 \mathrm{~km}, 200-300$ $\mathrm{km}$, and 160-200 km, respectively.

\subsection{Closed Field Lines}

Figure 8 presents the occurrence rate of closed field lines on the dayside (left column) and the nightside (right column). The occurrence rate is the number of spectra satisfying the criteria for this category (Table 1) divided by the total sample number. The rows from top to bottom show results for altitude ranges of $800-1000 \mathrm{~km}, 600-800 \mathrm{~km}, 400-600 \mathrm{~km}, 300-400 \mathrm{~km}$, 200-300 km, and 160-200 km, respectively. Bins with no value (white) occur when the total sample number is less than 50, which applies to Figures 9-11 as well. The gray contours are the modeled crustal magnetic field magnitude at $400 \mathrm{~km}$ [Morschhauser et al., 2014]. On the dayside, the most prominent trend is that the occurrence rate of closed field lines increases with decreasing altitude. This trend occurs over regions where the crustal field is relatively strong, as expected; however, it also occurs in the northern hemisphere, with closed field lines eventually dominating below 300-400 km (occurrence rate $>50 \%$ ), even over Hellas and Tharsis, the two most-weakly magnetized regions of the crust. In the lowest altitude range, magnetic field lines are actually less likely to be closed in some regions of the southern hemisphere, particularly near longitudes of $20^{\circ}$ and $300^{\circ}$ and poleward of $40^{\circ} \mathrm{S}$. Instead, these regions tend to have a fair amount of open field lines (see section 5.2).

For SZA > $110^{\circ}$, photoelectrons produced in the sunlit ionosphere can travel along closed magnetic field lines above the electron exobase and precipitate onto the nightside atmosphere [Xu et al., 2016b]. The occurrence rate for such a magnetic field configuration is presented in the right column of Figure 8. Overall, the rate is generally below $25 \%$ but exceeds $50 \%$ in some regions. The occurrence rate drops below $200 \mathrm{~km}$ altitude, possibly because of proximity to the electron exobase, where inelastic collisions degrade the photoelectron features. For altitudes above $600 \mathrm{~km}$, the maps are very similar, with regions of relatively high occurrence rates in the northern hemisphere and over the south polar region. Below $600 \mathrm{~km}$, low occurrence rates correspond to the strong crustal fields, suggesting that field lines tend to close more locally in these regions (see section 5.4). 

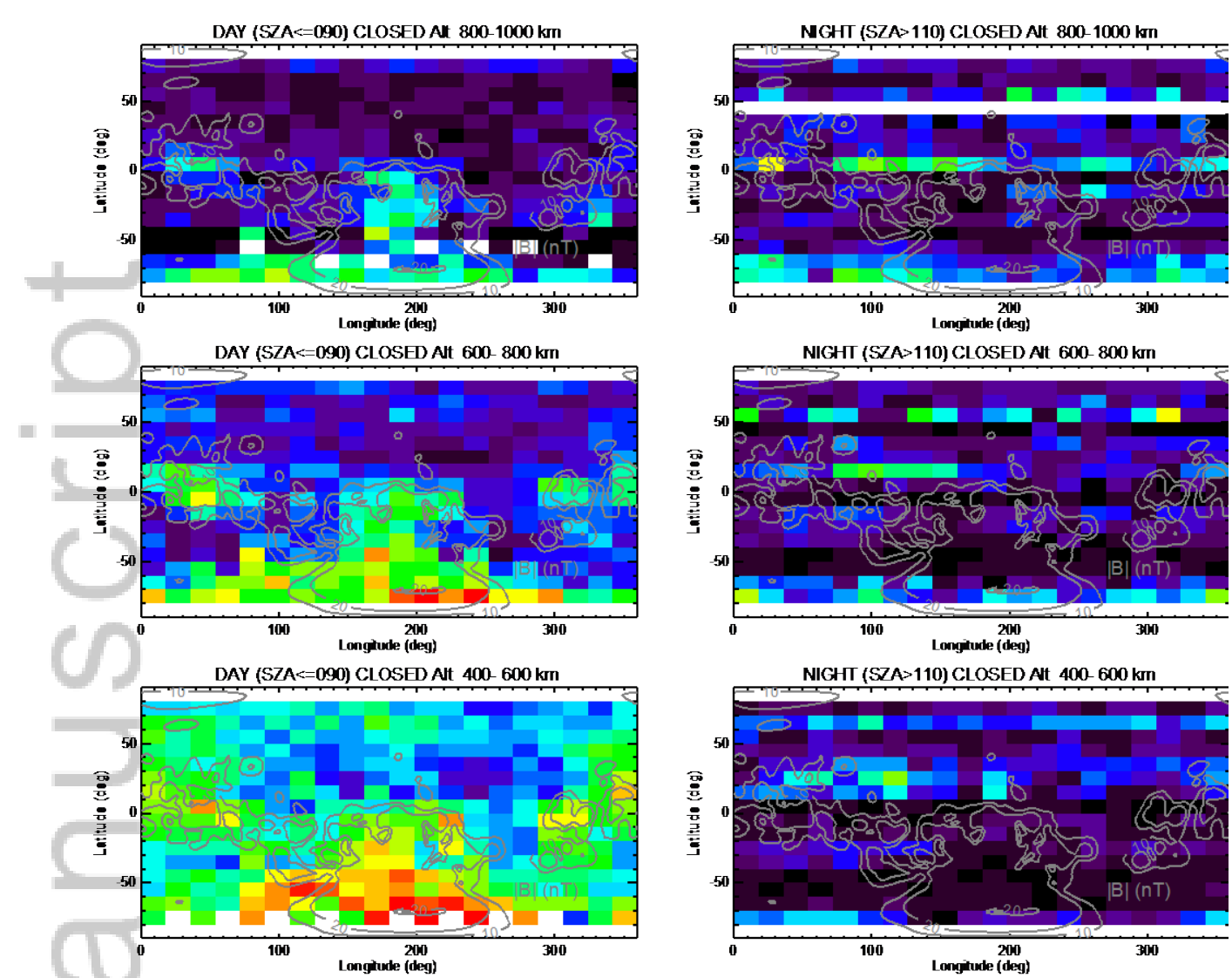

DAY $(S Z A<=090)$ CLOSED Alt $300-400 \mathrm{~km}$
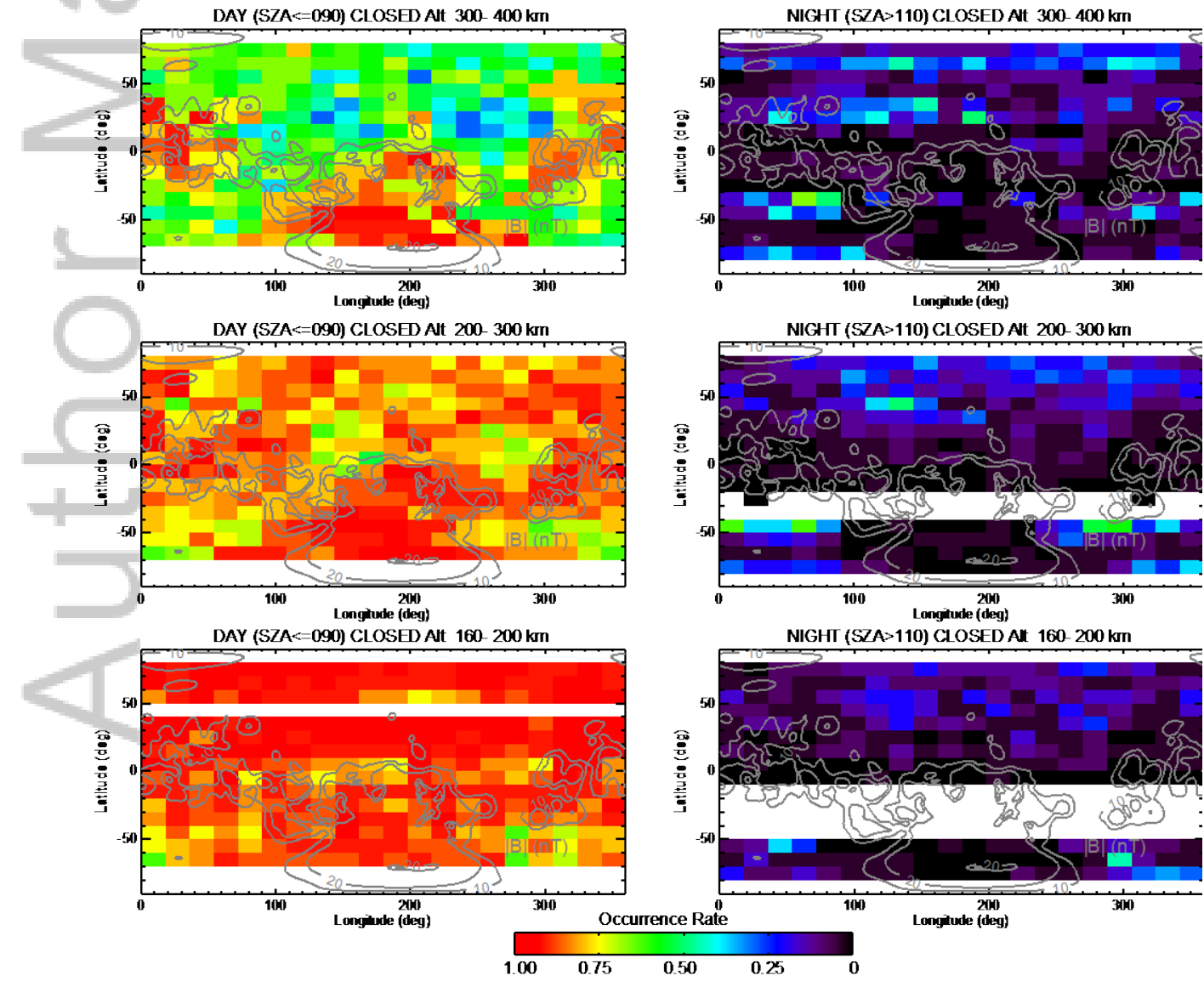

This article is protected by copyright. All rights reserved. 
Figure 8. The occurrence rate for closed magnetic field lines (color scale) based on pitch angle-resolved shape parameters is mapped in geographic longitude and latitude. Maps for the dayside $\left(\mathrm{SZA}<90^{\circ}\right.$ ) are shown in the left column, and nightside (SZA $>110^{\circ}$ ) maps are on the right. Representative field line geometries for the left (right) column is the purple (green) lines in Figure 2. Altitude ranges for each row are, from top to bottom, $800-1000$ km, $600-800$ km, 400-600 km, 300-400 km, 200-300 km, and 160-200 km, respectively. The gray contours are the modeled crustal magnetic field magnitude at 400 $\mathrm{km}$ [Morschhauser et al., 2014].

\subsection{Open Field Lines}

Maps for open field lines are shown in Figure 9, with the same format as Figure 8 but with a more compressed color scale. On the dayside, different trends can be seen over weak and strong crustal magnetic sources. Over weak sources (mostly in the northern hemisphere), the occurrence rate for open field lines is low above $800 \mathrm{~km}$ altitude, increases to $\sim 30-50 \%$ in the 300-400-km range, then falls significantly below $200 \mathrm{~km}$, where closed field lines dominate, as noted above. We will discuss this phenomenon in detail in section 6 . Finally, we note that one region of open field lines in the northern hemisphere $\left(50^{\circ}-60^{\circ} \mathrm{N}, 160^{\circ}-250^{\circ} \mathrm{E}\right)$ does map down to the 160-200-km altitude range. These open field lines may be associated with crustal sources near Arcadia (Figure. 1).

At high altitudes (> $800 \mathrm{~km}$ ) over strong sources, open field lines cluster over the strong crustal sources and are likely magnetic cusps that span large angular ranges at this high altitude. These cusps are expected to become narrower with decreasing altitudes as they approach the crustal sources; however, our longitude-latitude grid is too coarse to identify this effect. The high occurrence rate of open field lines over the weakly magnetized regions within $30^{\circ}$ of both poles, as well as on the nightside (right top panel), is probably due to open field lines originating from crustal sources and extending to high altitudes over the poles as they flare away from the MarsSun line. As the altitude decreases from 800 to $400 \mathrm{~km}$, open field lines become less common over strong crustal sources, as closed field lines become predominant. Below $200 \mathrm{~km}$, the occurrence rate of open field lines is low over most of the planet, except for Arcadia in the north, as noted above, and for two regions poleward of $40^{\circ} \mathrm{S}$ and centered near longitudes of $20^{\circ}$ and $300^{\circ}$. The two southern regions of open field lines may map to relatively weak crustal magnetic sources around the periphery of the Hellas and Argyre impact basins (Figure 1).

In the northern hemisphere at solar zenith angles greater than $110^{\circ}$, open field lines with access to the dayside ionosphere are rare below $200 \mathrm{~km}$ altitude (Figure 9, lower right panel). This is not unexpected, since open field lines originating in the dayside ionosphere, which 
become much more common above $200 \mathrm{~km}$ in the northern hemisphere, should flare away from the planet with increasing distance. This picture is confirmed by the first appearance of open field lines on the nightside in the $200-300-\mathrm{km}$ altitude bin, with a generally increasing occurrence rate at higher altitudes. The two regions of open field lines at high southern latitudes $\left(>60^{\circ} \mathrm{S}, 20^{\circ}-120^{\circ} \mathrm{E}\right.$ and $>60^{\circ} \mathrm{S}, 290^{\circ}-300^{\circ} \mathrm{E}$ ) are likely associated with strong crustal magnetic sources near the south pole. Interestingly, even for 800-1000 km in altitude, a region of low occurrence rates is seen and resembles the strong crustal regions (Figure 1), suggesting crustal control. While it might imply fewer open field lines resulting from interaction with IMF on the dayside when the strong southern crustal field located on the nightside, it is more likely that the underlying strong crustal fields on the nightside compel surrounding field lines away, which is an indirect proof of crustal control on the nightside extending beyond $1000 \mathrm{~km}$ [Brain et al., 2003].

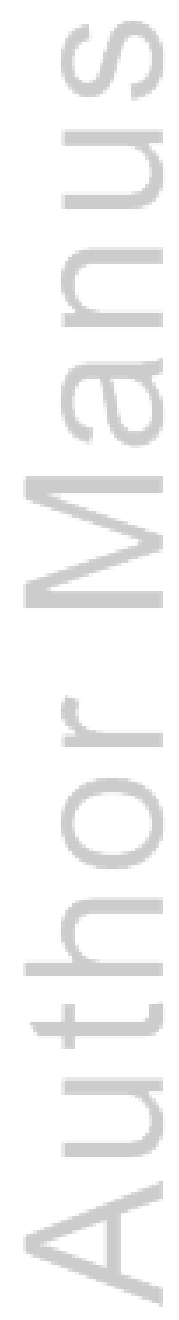



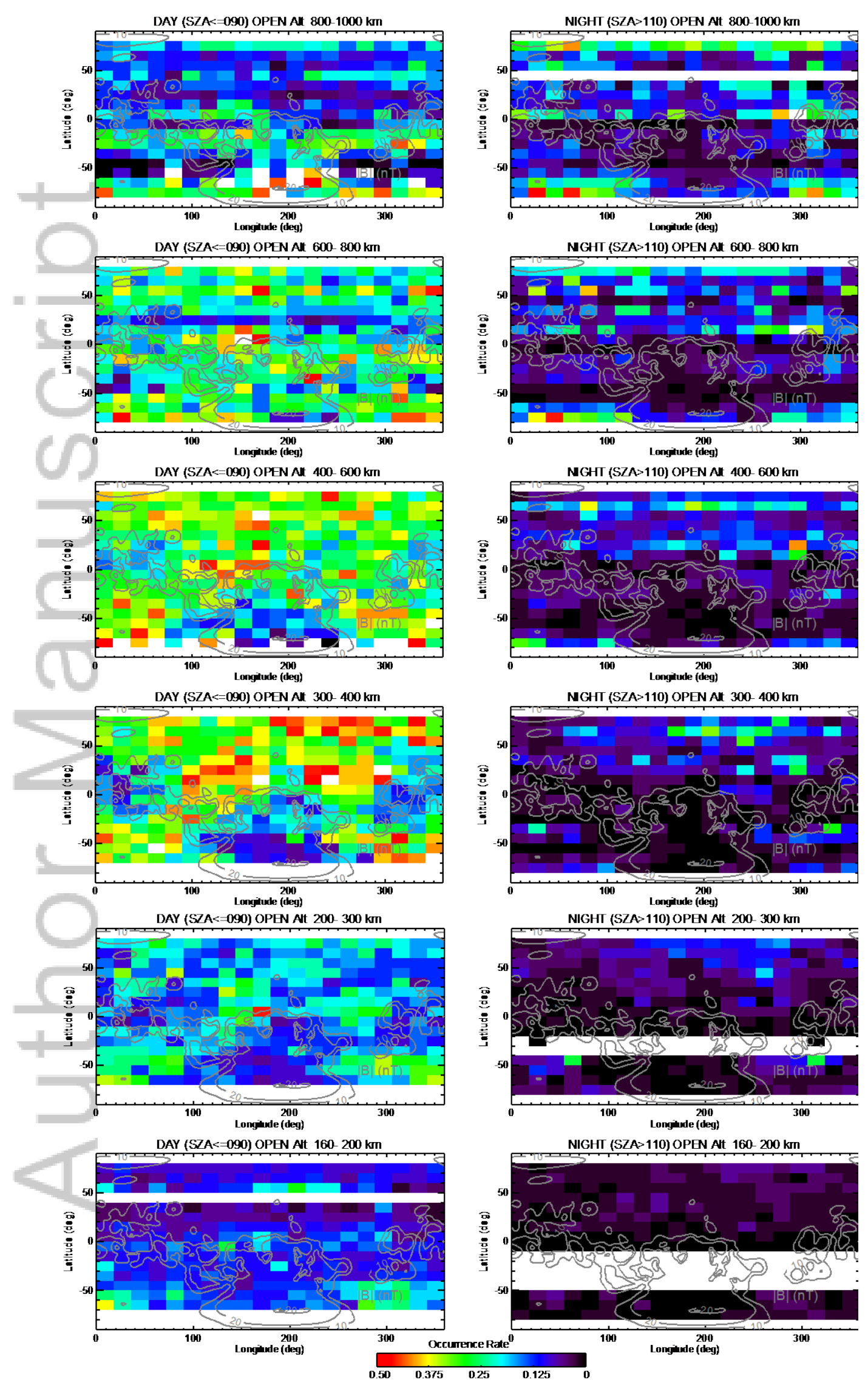

This article is protected by copyright. All rights reserved. 
Figure 9. The maps of open field lines, the same format as Figure 8 . The color scale is from 0 (black) to 0.5 (red). The representative field line geometry for both the left and right column is illustrated by the orange field lines in Figure 2. The difference is whether it was observed on the dayside or nightside.

\subsection{Draped Field Lines}

Figure 10 shows the occurrence rate for the draped IMF on the dayside (the left column) and the nightside (the right column). On the dayside, the occurrence rate increases with altitude, as expected. Below $400 \mathrm{~km}$ altitude, the field lines are mostly closed or open, so that few are draped. Beginning at $\sim 400 \mathrm{~km}$, IMF starts to drape over the northern hemisphere, with an occurrence rate of $20 \%-50 \%$ in the $400-600 \mathrm{~km}$ range, $>50 \%$ in the $600-800 \mathrm{~km}$ range, and $\sim 100 \%$ above $800 \mathrm{~km}$. In the south, strong crustal field regions can be discerned up to $1000 \mathrm{~km}$ with low draping occurrence rates and correspondingly high open and closed rates. A relatively high occurrence rate of draped fields above $800 \mathrm{~km}$ in the $40^{\circ}-60^{\circ} \mathrm{S}$ latitude range, but avoiding the longitudes of the strongest crustal sources from $160^{\circ}$ to $250^{\circ} \mathrm{E}$. This latitude band includes the Hellas and Argyre basins, which are the most-weakly magnetized regions of the southern hemisphere. Thus, it appears that draped IMF occupies a trough between strong crustal fields to the north and south. This is consistent with the analysis of MGS aerobraking magnetometer data [Brain et al. 2002], which indicates that the influence of the strongest crustal fields extends up to $\sim 1000 \mathrm{~km}$ altitude on the dayside.

On the nightside, the occurrence rate is mostly higher than on the dayside, because the classification of "draped" field lines here includes both draped IMF and open field lines with one footpoint on the nightside. Since draped field lines are expected to flare away from the MarsSun line with distance down the tail, the "draped" occurrence rate on the nightside is probably dominated by open field lines with footpoints in the nightside atmosphere. The low occurrence rates below $800 \mathrm{~km}$ over strong crustal sources correspond to the locations of voids (see below).

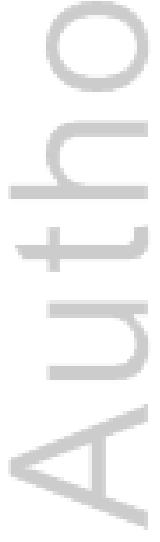



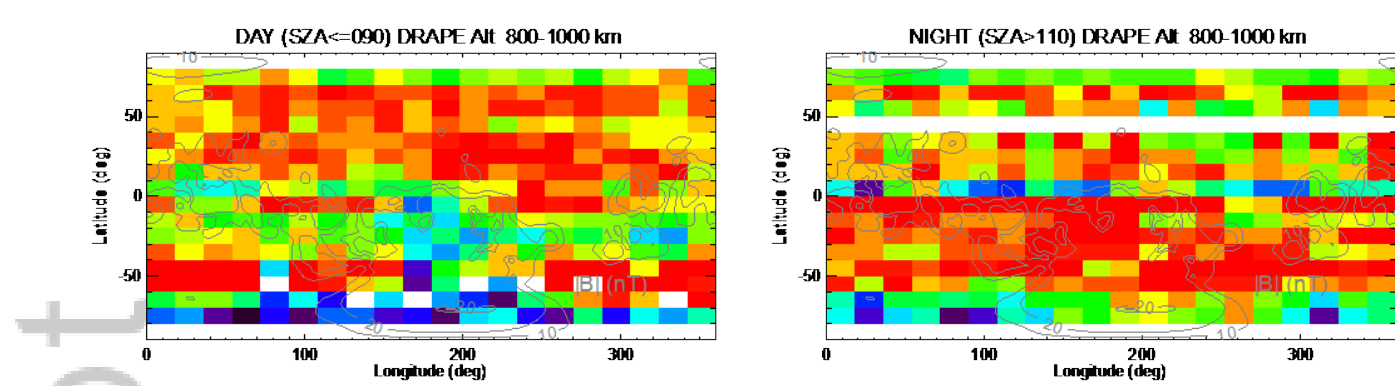

DAY (SZA $=090)$ DRAPE At $600-800 \mathrm{~km}$
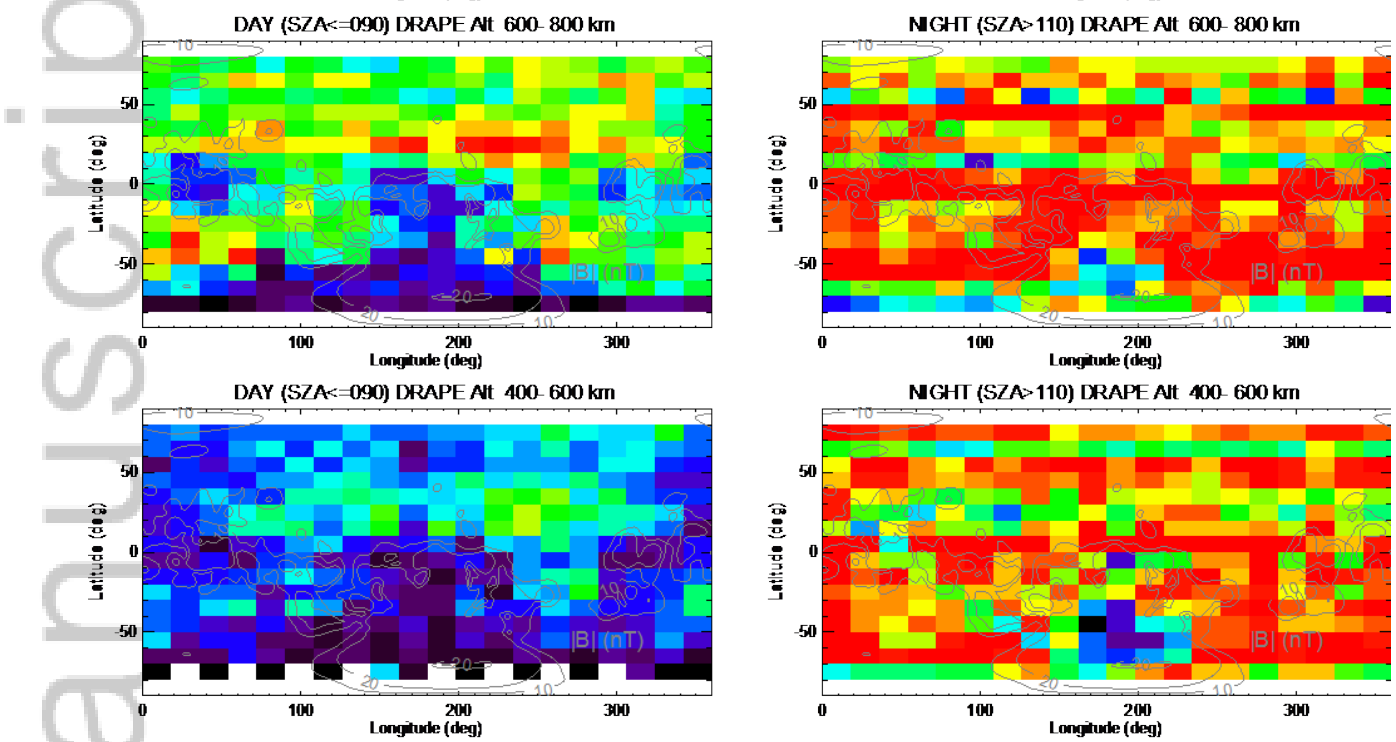

DAY (S/A $=090)$ DRAPE Alt $400-600 \mathrm{k}$

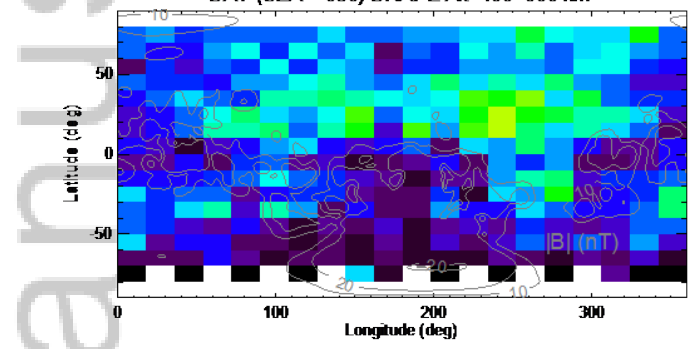

DAY (SZA $=090)$ DRAPE Al $300-400 \mathrm{~km}$
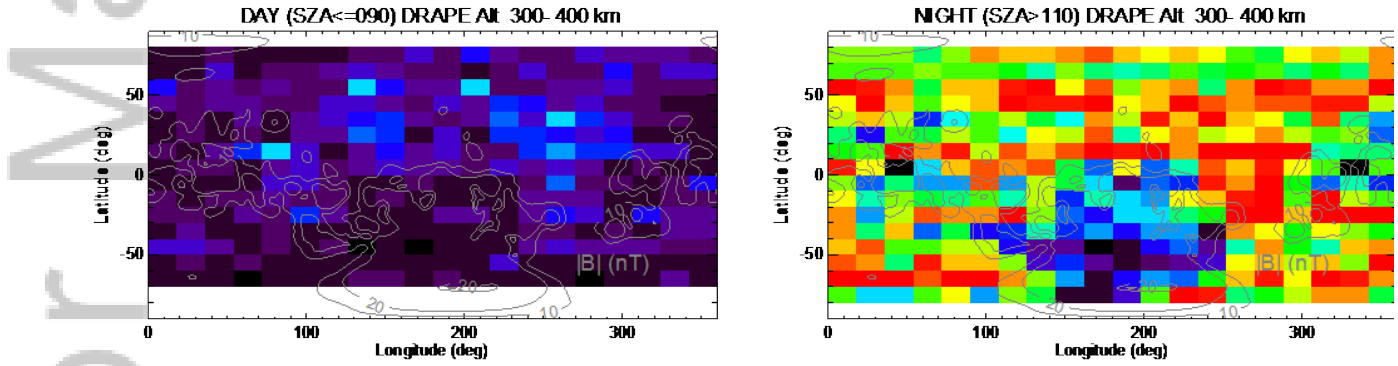

DAY (SZA $=090)$ DRAPE Alt $200-300 \mathrm{~km}$
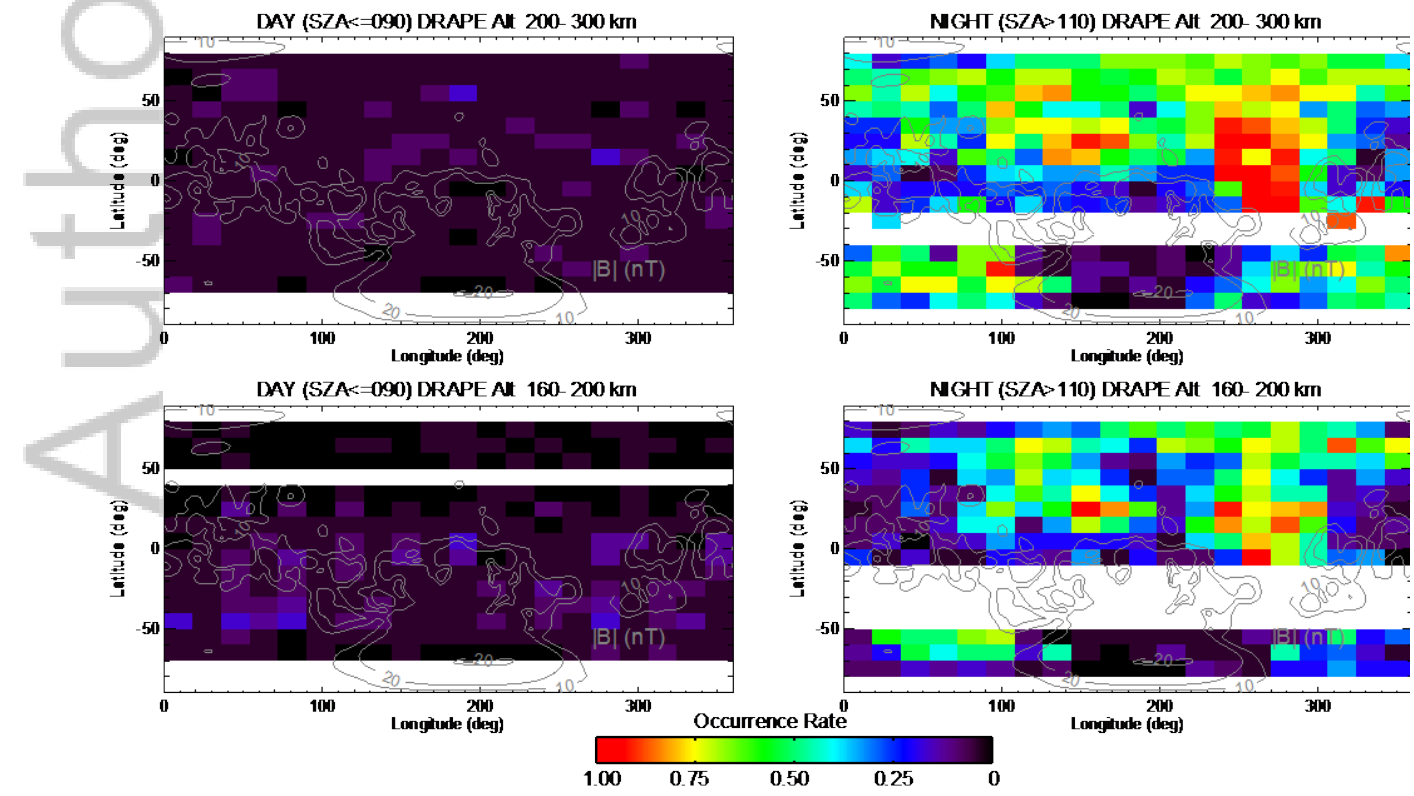

This article is protected by copyright. All rights reserved. 
Figure 10. The maps of draped field lines, the same format as Figure 8. The color scale is from 0 (black) to 1 (red). The right column includes both the draped IMF and open field lines attached to the nightside atmosphere (green lines in Figure 2).

\subsection{Voids}

Superthermal electron voids occur on closed crustal magnetic loops with both footpoints in the nightside atmosphere, and any trapped electron population has pitch-angle scattered into the loss cone or drifted out of the flux tube (Figure 11), so that the omnidirectional flux falls below our threshold (Table 1). Below $200 \mathrm{~km}$, the void occurrence rate is $>50 \%$ over most of the nightside, and nearly unity over the strongest crustal sources. The six altitude ranges reveal the three-dimensional morphology of the voids, which extend up to $\sim 1000 \mathrm{~km}$ over the stronger crustal sources. The longitude-latitude resolution of these maps is insufficient to resolve the narrow crustal magnetic cusps separating closed crustal loops of alternating polarity [Mitchell et al. 2007, Lillis et al. 2008]. These narrow cusps are readily seen in MAVEN time-series data (e.g., feature T2 in Figure 4).

The occurrence rate of closed crustal magnetic field lines is generally higher on the dayside (Figure 8, left panels) than on the nightside (Figure 11). One would expect strong crustal fields to be compressed on the dayside by the solar wind interaction, so that closed fields would extend to higher altitudes on the nightside. However, our definition of voids does not include closed crustal field loops with trapped populations, some of which could be identified as "draped" in Figure 10 (right side).

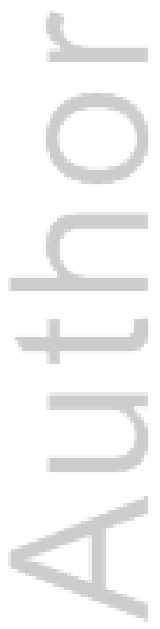

This article is protected by copyright. All rights reserved. 

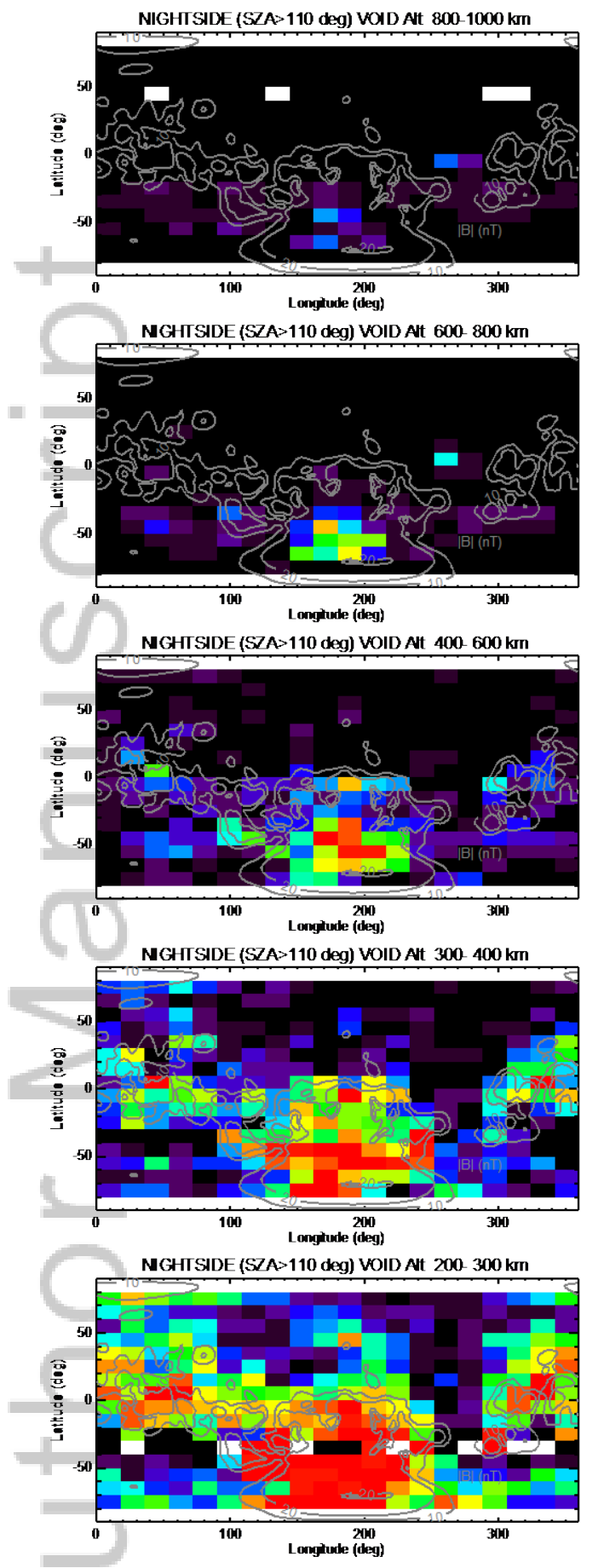

NIGHTSIDE (SZA>110 deg) VOID Nt $160-200 \mathrm{~km}$

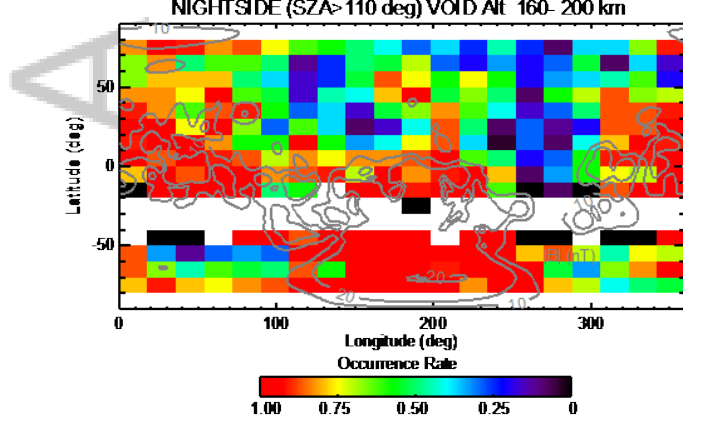

This article is protected by copyright. All rights reserved. 
Figure 11. The maps of voids on the nightside $\left(\mathrm{SZA}>110^{\circ}\right)$. The color stands for the occurrence rate. The representative field line geometry is the black lines in Figure 2. From top to bottom, each panel is for the altitude range of $800-1000 \mathrm{~km}, 600-800 \mathrm{~km}$, $400-600 \mathrm{~km}, 300-400 \mathrm{~km}, 200-300 \mathrm{~km}$, and 160-200 km, respectively. The color scale is from 0 (black) to 1 (red). The gray contours are the modeled crustal magnetic field magnitude at $400 \mathrm{~km}$ [Morschhauser et al., 2014].

\section{Discussion}

This study provides the first three-dimensional map of magnetic field topology from the electron exobase to $1000 \mathrm{~km}$ altitude. The electron exobase, which defines the lowest altitude at which electron energy-pitch angle distributions can be used to infer magnetic topology, is not at a fixed altitude, but instead depends on the atmospheric density profile and the orientation of the magnetic field with respect to vertical. The lowest altitude bin in this study extends down to 160 $\mathrm{km}$, which is the electron exobase altitude based on electron transport calculations along a vertical magnetic field line. When the field line is not vertical, the electron exobase occurs at a higher altitude. Figure 12a shows how the "collisional depth" $\tau$ [Xu et al., 2016a] varies with magnetic elevation angle, or dip angle. The collisional depth $\tau(\mathrm{h})$, similar to the optical depth, is defined as the integral of the product of neutral or thermal plasma density and collision cross sections along a field line, from a high altitude where collisions are negligible to a given altitude h. For this calculation, we include electron-neutral, electron-electron, and electron-ion collisions (see Equation 2 of Xu et al. [2016a]). This dimensionless quantity approximates the likelihood that an electron will suffer a collision as it travels from the top of a field line (here is $400 \mathrm{~km}$ ) to a given altitude, or vice versa. The electron exobase is defined to be the altitude where $\tau=1$, i.e., below $185 \mathrm{~km}$ for magnetic elevation angles $>10^{\circ}$ and $\sim 220 \mathrm{~km}$ for elevation angles $\sim 1^{\circ}$. These

values are for 20-eV electrons, but Xu et al. [2016a] showed the exobase varies by less than $5 \mathrm{~km}$ from 20 to $200 \mathrm{eV}$.

The neutral and plasma density profiles used for this calculation are taken from the simulation results of the Mars Thermospheric General Circulation Model (MTGCM) [Bougher et al., 1999, 2000], the same as Xu et al. [2016a], run at a solar longitude (Ls) of $90^{\circ}$ and with an Earth $\mathrm{F}_{10.7}$ of $100 \mathrm{sfu}$ ( $43 \mathrm{sfu}$ at Mars). We assume the density profiles are the same as $\mathrm{SZA}=0^{\circ}$ along the path for all the elevation angles, which results in an overestimation for the exobase altitudes in Figure 12a. Figure 12b illustrates the average absolute magnetic elevation angle measured by MAVEN MAG at 160-200 km on the dayside over the same time period as the electron data. For most of the regions, the average elevation angle is greater than $20^{\circ}$, which 
corresponds to an electron exobase of $\sim 185 \mathrm{~km}$. The higher exobase altitude for more horizontal fields can affect the results in the lowest altitude range, especially over the northern weak crustal regions, but our methodology should be robust above $200 \mathrm{~km}$. This is also supported by the fact that the shape parameters pick up significant amount of open field lines for 200-300 km altitude range, which means it is distinguishable between solar wind electrons and photoelectrons above $200 \mathrm{~km}$.
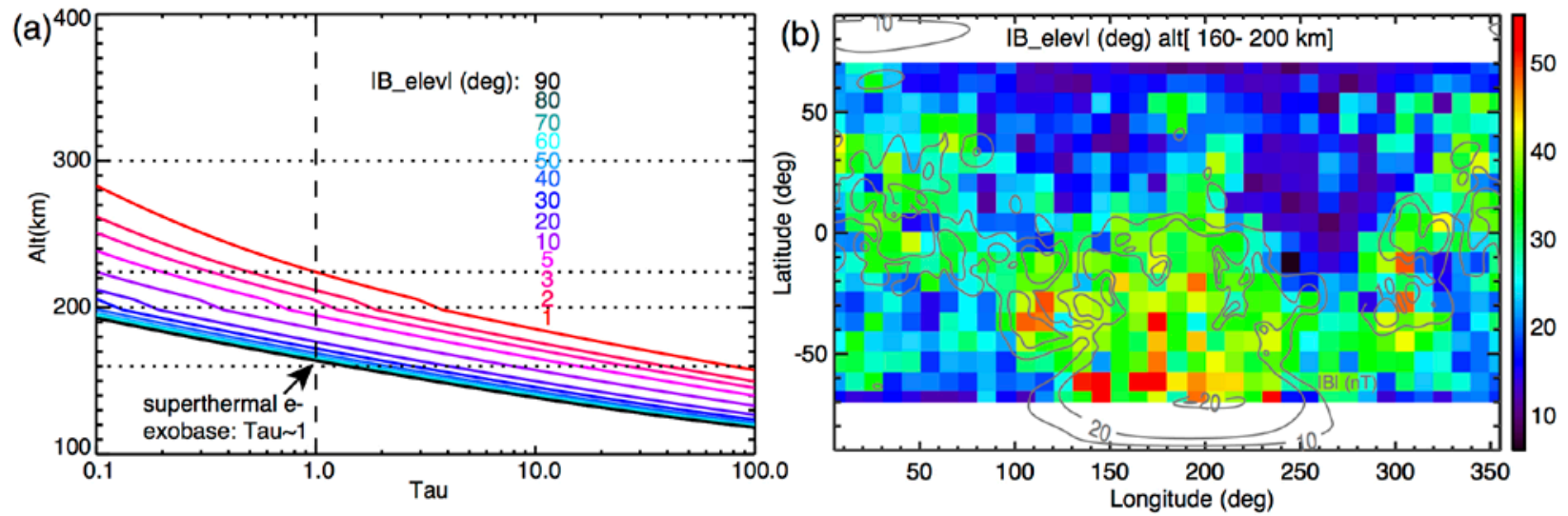

Figure 12. (a) Tau against altitude for different magnetic elevation angles. The vertical dashed line indicates $\mathrm{Tau}=1$, where the superthermal electron exobase is. (b) The average absolute magnetic elevation angle measured by MAVEN MAG at 160-200 km on the dayside. The gray contours are the modeled crustal magnetic field magnitude at $400 \mathrm{~km}$ [Morschhauser et al., 2014].

Based on extensive observations at $400 \mathrm{~km}$ altitude by the MGS MAG/ER, the magnetic topology at $2 \mathrm{pm}$ over the weakest crustal magnetic field regions in the northern hemisphere $\left(50^{\circ}-60^{\circ} \mathrm{N}\right)$ was found to be dominated by draped IMF [Brain et al. 2006]. Our maps show that this is in fact the lowest altitude where draped fields are significant in this region. Closed field lines are found to be dominant below $400 \mathrm{~km}$, even over the weak crustal regions in the northern hemisphere. Although the electron exobase over these weak crustal regions intrudes into the lowest altitude bin, the occurrence rate for closed field lines increases from $50 \%$ in the 300-400 $\mathrm{km}$ altitude bin to $>75 \%$ in the $200-300-\mathrm{km}$ bin, which indicates that closed field lines become increasingly prevalent at low altitudes. Although we have no way of determining field topology below the electron exobase, one possibility is that these closed field lines are of crustal origin. In this case, the closed loops either connect two distant, previously mapped crustal sources (for a possible example, see Xu et al., 2016b), or they are associated with more local, unmapped sources. A second possibility is that an open or draped field line could have a perturbation such that a segment of the line starts below the electron exobase, rises up to the spacecraft altitude, then dips below the electron exobase again. If this is the main explanation, then these perturbations must have a vertical amplitudes of $\sim 100$ 's of $\mathrm{km}$, be widespread and occur much of 
the time. Note that over the northern weak regions, the elevation angles are small in Figure $12 b$, which seemingly implies these field lines are more likely to be draped IMF. However, these can also be large closed field lines connecting distant crustal sources so that they are mostly horizontal over the weak regions. Moreover, over the weak regions, if crustal fields are locally closed, it is likely we are observing the top of the field lines, which tend to be horizontal as well. We can also use the maps to examine the photoelectron boundary (PEB) [Mitchell et al., 2000]. If we define the PEB as the altitude at which there is a 50\% probability of observing closed field lines, then we can find that the PEB is located at 300-400 km in the north and $\sim 600 \mathrm{~km}$ in the south.

The open field line occurrence rate decreases dramatically from 200-300 km range to 160-200 km range over the north. This could be explained by one or more of the following explanations. First, it could be that the electron exobase over weak crustal sources (more horizontal magnetic fields) intrudes into the lowest altitude bin enough to bias the probability. Another contributing factor could be that the open field lines converge with decreasing altitude, thus spanning a smaller solid angle and becoming less likely to be observed. The third contributing factor could be that open field lines observed in the northern hemisphere above 200 $\mathrm{km}$ intersect the electron exobase at some distant location, most likely near the equator and/or in the south, where strong crustal magnetic sources are present. For such a scenario, open field lines originate from footpoints above strong equatorial crustal sources and become more horizontal as they wrap around the planet and extend down the tail. These field lines pass over the northern hemisphere at higher altitudes with a more horizontal orientation. The presence of open field lines, possibly associated with strong crustal sources to the south, beneath the draped IMF would provide an explanation for the asymmetry in the draping direction inferred from MGS observations at $400 \mathrm{~km}$ altitude [Brain et al. 2006]. The draping pattern is possibly formed by open field lines draping over the northern hemisphere, resulting from the solar wind interaction with crustal fields at low altitudes. This suggests the presence of topological "layers" over the northern hemisphere: closed field lines below $200 \mathrm{~km}$, open field lines with footpoints at lower latitudes that pass over the northern hemisphere or closed field lines connecting distant crustal sources for $200-400 \mathrm{~km}$, and draped IMF above $400 \mathrm{~km}$. This suggests that the influence of crustal fields extends over the entire planet, preventing IMF penetration below $\sim 400 \mathrm{~km}$ under the typical upstream conditions.

Consider the occurrence rate of low-altitude $(160-200 \mathrm{~km})$ closed field lines over the two most weakly magnetized regions in the northern hemisphere, the Utopia basin and the Tharsis rise. When both footpoints are on the nightside (superthermal electron voids), the occurrence rate ranges from $10 \%$ to $50 \%$. When both footpoints are on the dayside (photoelectrons in both directions), the occurrence rate is close to $100 \%$. One possibility is that collisions with the 
neutral atmosphere in the lower part of this altitude range are limiting our ability to infer topology because electron motion is dominated by collisions rather than by the magnetic field. On the nightside, voids would be caused by collisions and would occur regardless of the magnetic field topology. On the dayside, the spacecraft would be embedded in the ionospheric production region, and photoelectrons would be incident from all directions.

-The observations shown in Figures 5 and 6 provide a test of this possibility. On this date, periapsis occurs in darkness at an altitude of $155 \mathrm{~km}$ (Figure 5, T2). At this time, the shape parameter analysis indicates a "draped" topology (Figure 6, center); however, an alternative and more likely interpretation is an open field line with one footpoint on the night hemisphere. The parallel population (red spectrum) is solar wind electrons precipitating onto the atmosphere, and the anti-parallel population (blue spectrum) is backscattered electron flux [e.g. Collison et al., 2016]. Both spectra show evidence for significant modification by collisions, including a reduced flux at all energies and a change in the spectral shape compared with solar wind spectra measured at higher altitudes (Fig. 6, T3). On either side of the precipitation region at T2, the spacecraft passes through superthermal electron voids at nearly the same altitude. Without a precipitating solar wind flux, the electron populations at these locations have more completely thermalized, with only a residual superthermal population peaking near $7 \mathrm{eV}$. The intermittent occurrence of a precipitating flux indicates that magnetic topology still plays an important role at these altitudes. Thus, we can confidently interpret voids observed above $160 \mathrm{~km}$ as topological, that is closed field lines with both footpoints on the nightside.

Since the atmospheric scale height is smaller on the nightside, the electron exobase should occur at a lower altitude than shown in Figure 12a. Significant superthermal electron depletions caused by collisions with the neutral atmosphere occur below the electron exobase. For example, from the middle panel of Figure 6, we see that the electron flux does not reach the threshold of a void (by our definition) even at the periapsis altitude of $155 \mathrm{~km}$. The occurrence rate of night-time voids over Utopia and Tharsis is significant (10-50\%) but much lower than the $\sim 100 \%$ occurrence rate of closed field lines during the day. This difference might be because superthermal electron voids represent only a subset of closed field lines on the night side. There could also be closed field lines with electrons mirroring above the collisional atmosphere, which would likely be categorized as "draped" field lines in our scheme (solar wind electrons in both directions). Another possibility is that there might in fact be fewer closed field lines over Utopia and Tharsis on the nightside, if it is more likely for these regions to reconnect with the solar wind in the tail than on the dayside. Now, consider the two-aforementioned possible closed geometries over the weak crustal regions, draped IMF intersecting the collisional atmosphere twice and closed field lines connecting to crustal source. For the first scenario, it might be that 
fewer draped IMFs connect twice to the dense atmosphere as they move towards nightside, only intersecting once or not at all. For the closed field lines connecting distant crustal source, i.e. the northern hemisphere layering, crustal fields on the dayside, opened by reconnection, tend to lay over the weak regions, either just as open field lines or closed up with distant crustal sources. In contrast, on the nightside, crustal fields tend to stretch to down tail and reconnect with solar wind fields, no longer "protecting" the weak regions so that it is easier for IMF to penetrate into low altitudes and form open field lines.

On the nightside, the northern hemisphere is found to be mostly "draped" according to our selection criteria (Table 1). However, as noted above, our criteria for nightside "draped" fields also include open field lines with one footpoint in the nightside atmosphere. MGS observations show that open field lines are common in the northern hemisphere at 2 am local time [Mitchell et al. 2007, Lillis et al. 2008]. In contrast, electron voids dominate over strong crustal fields in the southern hemisphere and near the equator at low altitudes. The detailed structure of these void regions (see Figure 2 of Mitchell et al. 2005) is unresolved by our longitude-latitude grid. Such closed field lines prevent superthermal electron precipitation, which is the main source of ionization on the nightside, as well as day-to-night transport above the exobase. Where these closed field lines map to the electron exobase, the ionosphere must be dominated by long-lived ions, such as $\mathrm{NO}^{+}$, that can survive during the Martian night [GonzálezGalindo et al. 2013]. In the north, cross-terminator closed field lines (occurrence rate < 25\%) can also provide a source of superthermal electrons to the deep nightside [Xu et al., 2016b].

All of the magnetic field topologies inferred in this study are present in MHD simulations (e.g., Figure 2). For example, the low-altitude cross-terminator closed field lines are common in both observations and simulations of the northern hemisphere (see also Xu et al., 2016b). Also, the absence of draped field lines below $300 \mathrm{~km}$ altitude on the dayside is shown in both model predictions and observations (the bottom left panel of Figure 10). Thus, this technique can be used to validate simulation results.

Open field lines can intersect the electron exobase on either the dayside or the nightside. Open field lines connected to the dayside ionosphere provides a path for ion outflow, and are thus potentially important for ion escape. These lines occur $<50 \%$ of the time on the dayside and are generally confined to high latitudes on the nightside, with a higher occurrence rate in the northern hemisphere. Open field lines connected to the nightside atmosphere allow precipitation of solar wind electrons and (episodically) SEP (Solar Energetic Particle) electrons, which causes heating, excitation, and ionization, and occasionally observable auroral emissions [e.g., Schneider et al., 2015]. These field lines are common on the nightside (e.g., Mitchell et al. 2005) 
but are identified as "draped" in our study (Fig. 10, right side), which is based on the shapes of electron energy spectra and not the presence of one-sided loss cones.

\section{Conclusions and Future Work}

MAVEN is the first mission to systematically sample the Mars plasma environment down to altitudes of $\sim 150 \mathrm{~km}$ over wide ranges of longitude, latitude, local time, and solar zenith angle. We can readily distinguish ionospheric primary photoelectrons from solar wind electrons and with pitch angle resolved shape parameters we deduce the magnetic topology from the electron exobase to $1000 \mathrm{~km}$ altitude. For the first time, we are able to determine the topology below 400 $\mathrm{km}$. This study finds that the sunlit hemisphere below $400 \mathrm{~km}$ altitude is dominated by closed field lines, even in the northern hemisphere. These maps combined illustrate how the magnetic topology evolves in three dimensions, in particular how one topology connects to another and how crustal control can happen over a large distance. Overall, the results are consistent with many findings from MGS and also qualitatively agree with MHD results. Open field lines attached to the dayside ionosphere can be mapped out by this methodology, which is a key piece to understand ion outflow, and those intersecting the nightside ionosphere allow energetic electron (solar wind electrons and SEP electrons) precipitations, critical to understand the nightside ionosphere dynamics.

This study focuses only on the magnetic topology's dependence on the geographic latitude, longitude, and altitude. One future work would analyze how the topology changes with upstream conditions and the orientation of the crustal magnetic fields with respect to the MarsSun line. Our methodology can also be used to analyze the tail magnetic topology as well, especially in the flanks. In addition, the observations qualitatively confirm the predictions of field line types from the multispecies Mars-MHD model. A direct data-model comparison can be performed in the future to further our understanding of the Martian plasma environment from both observational and theoretical points of views.

\section{Acknowledgments and Data}

This work was supported by the NASA Mars Scout Program. Work at Michigan was supported by NASA R\&A grants. The MAVEN data used in this study are available through Planetary Data System. The BATS-R-US code is publicly available from http://csem. engin.umich.edu/tools/swmf. For distribution of the MHD magnetic field line tracing results used in this study, contact X. Fang (Xiaohua.Fang@lasp.colorado.edu). 


\section{References:}

Acuna, M., et al. (1992), Mars observer magnetic fields investigation, Journal of Geophysical Research: Planets (1991-2012), 97(E5), 7799-7814.

Acuna, M., et al. (1998), Magnetic field and plasma observations at Mars: Initial results of the Mars Global Surveyor mission, Science, 279 (5357), 1676-1680.

Banks, P., and A. Nagy (1970), Concerning the influence of elastic scattering upon photoelectron transport and escape, Journal of Geophysical Research, 75(10), 1902-1910.

Bertaux, J.-L., F. Leblanc, O. Witasse, E. Quemerais, J. Lilensten, S. Stern, B. Sandel, and O. Korablev (2005), Discovery of an aurora on Mars, Nature, 435(7043), 790-794.

Bougher, S. W., S. Engel, R. Roble, and B. Foster (1999), Comparative terrestrial planet thermospheres: 2. Solar cycle variation of global structure and winds at equinox, J. Geophys. Res., 104(E7), 16,591-16,611.

Bougher, S. W., S. Engel, R. Roble, and B. Foster (2000), Comparative terrestrial planet thermospheres: 3. Solar cycle variation of global structure and winds at solstices, J. Geophys. Res., 105(E7), 17,669-17,692.

Brain, D., F. Bagenal, M. Acuna, and J. Connerney (2003), Martian magnetic morphology: Contributions from the solar wind and crust, Journal of Geophysical Research: Space Physics (1978-2012), 108(A12).

Brain, D., J. Halekas, R. Lillis, D. Mitchell, R. Lin, and D. Crider (2005), Variability of the altitude of the Martian sheath, Geophys. Res. Lett., 32, L18203, doi:10.1029/2005GL023126.

Brain, D., J. Halekas, L. Peticolas, R. Lin, J. Luhmann, D. Mitchell, G. Delory, S. Bougher, M. Acun a, and H. Rème (2006), On the origin of aurorae on Mars, Geophys. Res. Lett., 33, L01201, doi:10.1029/2005GL024782.

Brain, D., R. Lillis, D. Mitchell, J. Halekas, and R. Lin (2007), Electron pitch an- gle distributions as indicators of magnetic field topology near mars, Journal of Geophysical Research: Space Physics (1978-2012), 112(A9).

Collinson, G., D. Mitchell, A. Glocer, J. Grebowsky, W. K. Peterson, J. Connerney, L. Andersson, J. Espley, C. Mazelle, J.-A. Sauvaud, et al. (2015), Electric Mars: The first direct measurement of an upper limit for the Martian “polar wind” electric potential, Geophys. Res. Lett., 42,9128-9134, doi:10.1002/2015GL065084.

Collinson, G., et al. (2016), Electric Mars: A large trans-terminator electric potential drop on closed magnetic field lines above Utopia Planitia, J. Geophys. Res. Space Physics, 121, doi:10.1002/2016JA023589.

Connerney, J., M. Acuñ a, N. Ness, G. Kletetschka, D. Mitchell, R. Lin, and H. Reme (2005), Tectonic implications of mars crustal magnetism, Proceedings of the na- tional Academy of Sciences of the United States of America, 102(42), 14,970- 14,975. 
Connerney, J. E. P., J. Espley, P. Lawton, S. Murphy, J. Odom, R. Oliversen, and D. Sheppard (2015). "The MAVEN magnetic field investigation." Space Science Reviews 195, no. 1-4: 257-291.

Fang, X., Y. Ma, D. Brain, Y. Dong, and R. Lillis (2015), Control of Mars global atmospheric loss by the continuous rotation of the crustal magnetic field: A time-dependent MHD study, J. Geophys. Res. Space Physics, 120, 10,926-10,944, doi:10.1002/2015JA021605.

Fillingim, M. O., L. M. Peticolas, R. J. Lillis, D. A. Brain, J. S. Halekas, D. L. Mitchell, R. P. Lin, D. Lummerzheim, S. W. Bougher, and D. L. Kirchner (2007), Model cal- culations of electron precipitation induced ionization patches on the nightside of Mars, Geophysical Research Letters, 34, L12101, doi:10.1029/2007GL029986.

Fillingim, M., L. Peticolas, R. Lillis, D. Brain, J. Halekas, D. Lummerzheim, and S. Bougher (2010), Localized ionization patches in the nighttime ionosphere of mars and their electrodynamic consequences, Icarus, 206(1), $112-119$.

Frahm, R., J. Winningham, J. Sharber, J. Scherrer, S. Jeffers, A. Coates, D. Linder, D. Kataria, R. Lundin, S. Barabash, et al. (2006), Carbon dioxide photoelectron energy peaks at mars, Icarus, 182(2), 371-382.

Frahm, J.R. Sharber, J.D. Winningham, R. Link, M.W. Liemohn, J.U. Kozyra, A.J. Coates, D.R. Linder, S. Barabash, R. Lundin, A. Fedorov (2010), Estimation of the escape of photoelectrons from Mars in 2004 liberated by the ionization of carbon dioxide and atomic oxygen, Icarus, Volume 206, Issue 1, March 2010, Pages 50-63, ISSN 0019-1035, http://dx.doi.org/10.1016/j.icarus.2009.03.024.

Ganguli, Supriya B. "The polar wind." Reviews of Geophysics 34.3 (1996): 311-348.

Glocer, A., G. Tóth, T. Gombosi, and D. Welling (2009), Modeling ionospheric outflows and their impact on the magnetosphere, initial results, J. Geophys. Res., 114, A05216, doi:10.1029/2009JA014053.

González-Galindo, F., J.-Y. Chaufray, M. A. López-Valverde, G. Gilli, F. Forget, F. Leblanc,R. Modolo, S. Hess, and M. Yagi (2013), Three-dimensional Martian ionosphere model: I. The photochemical ionosphere below 180 km, J. Geophys. Res. Planets, 118, 2105-2123, doi:10.1002/jgre.20150.

Harada, Y., et al. (2016), MAVEN observations of electron-induced whistler mode waves in the Martian magnetosphere, J. Geophys. Res. Space Physics, 121, 9717-9731, doi:10.1002/2016JA023194.

Harnett, E. M., and R. M. Winglee (2005), Three-dimensional fluid simulations of plasma asymmetries in the Martian magnetotail caused by the magnetic anomalies, Journal of Geophysical Research: Space Physics, 110(A7), n/a-n/a, doi: 10.1029/2003JA010315.

Jakosky, Bruce M., et al. "The Mars atmosphere and volatile evolution (MAVEN) mission." Space Science Reviews 195.1-4 (2015): 3-48. 
Khazanov, G. V., M. W. Liemohn, and T. E. Moore (1997), Photoelectron effects on the self-consistent potential in the collisionless polar wind, J. Geophys. Res., 102, 7509.

Krymskii, A., T. Breus, N. Ness, M. Acun a, J. Connerney, D. Crider, D. Mitchell, and S. Bauer (2002), Structure of the magnetic field fluxes connected with crustal magnetization and topside ionosphere at Mars, J. Geophys. Res., 107(A9), 1245, doi:10.1029/2001JA000239.

Krymskii, A., N. Ness, D. Crider, T. Breus, M. Acun a, and D. Hinson (2004), Solar wind interaction with the ionosphere/atmosphere and crustal magnetic fields at Mars: Mars global surveyor magnetometer/electron reflectometer, radio science, and accelerometer data, J. Geophys. Res., 109, A11306, doi:10.1029/2004JA010420.

Leblanc, F., et al. (2008), Observations of aurorae by SPICAM ultraviolet spectrograph on board Mars Express: Simultaneous ASPERA-3 and MARSIS measurements, J. Geophys. Res., 113, A08311, doi:10.1029/2008JA013033.

Liemohn, M. W., D. L. Mitchell, A. F. Nagy, J. L. Fox, T. W. Reimer, and Y. Ma (2003), Comparisons of electron fluxes measured in the crustal fields at mars by the mgs magnetometer/electron reflectometer instrument with $\mathrm{a} b$ field-dependent transport code, Journal of Geophysical Research, 108(E12), 5134.

Liemohn, Michael W., et al (2006a). "Numerical interpretation of high-altitude photoelectron observations." Icarus 182.2: 383-395.

Liemohn, M. W., Y. Ma, R. A. Frahm, X. Fang, J. U. Kozyra, A. F. Nagy, J. D. Winningham, J. R. Sharber, S. Barabash, and R. Lundin (2006b), Mars Global MHD Predictions of Magnetic Connectivity Between the Dayside Ionosphere and the Magnetospheric Flanks, Space Science Reviews, 126, 63-76, doi:10.1007/s11214006-9116-8.

Liemohn, M., Y. Ma, A. Nagy, J. Kozyra, J. Winningham, R. Frahm, J. Sharber, S. Barabash, and R. Lundin (2007), Numerical modeling of the magnetic topology near Mars auroral observations, Geophysical Research Letters, 34 (24).

Lillis, R. J., D. L. Mitchell, R. P. Lin, J. E. P. Connerney, and M. H. Acuña (2004), Mapping crustal magnetic fields at Mars using electron reflectometry, Geophys. Res. Lett., 31, L15702, doi:10.1029/2004GL020189.

Lillis, R. J., D. L. Mitchell, R. P. Lin, and M. H. Acuña (2008), Electron reflectometry in the Martian atmosphere, Icarus, 194, 544-561, doi:10.1016/j.icarus.2007.09.030.

Lillis, R. J., M. O. Fillingim, L. M. Peticolas, D. A. Brain, R. P. Lin, and S. W. Bougher (2009), Nightside ionosphere of Mars: Modeling the effects of crustal magnetic fields and electron pitch angle distributions on electron impact ionization, Journal of Geophysical Research (Planets), 114, E11009, doi:10.1029/2009JE003379. 
Lillis, R. J., and D. A. Brain (2013), Nightside electron precipitation at mars: Geographic variability and dependence on solar wind conditions, J. Geophys. Res. Space Physics, 118, 3546-3556, doi:10.1002/jgra.50171.

Lillis, Robert J., David A. Brain, Stephen W. Bougher, François Leblanc, Janet G. Luhmann, Bruce M. Jakosky, Ronan Modolo et al (2015). "Characterizing atmospheric escape from Mars today and through time, with MAVEN." Space Science Reviews 195, no. 1-4: 357-422.

Ma, Y., A. F. Nagy, I. V. Sokolov, and K. C. Hansen (2004), Three-dimensional, multispecies, high spatial resolution MHD studies of the solar wind interaction with Mars, J. Geophys. Res.,109, A07211, doi: $10.1029 / 2003 J A 010367$.

Ma, Y. J., X. Fang, A. F. Nagy, C. T. Russell, and G. Toth (2014a), Martian ionospheric responses to dynamic pressure enhancements in the solar wind, Journal of Geophysical Research: Space Physics, 119(2), 1272-1286, doi: 10.1002/2013JA019402.

Ma, Y., X. Fang, C. T. Russell, A. F. Nagy, G. Toth, J. G. Luhmann, D. A. Brain, and C. Dong (2014b), Effects of crustal field rotation on the solar wind plasma interaction with Mars,Geophys. Res. Lett., 41, 6563-6569, doi:10.1002/2014GL060785.

Mitchell, D., R. Lin, H. Reme, D. Crider, P. Cloutier, J. Connerney, M. Acuña, and N. Ness (2000), Oxygen auger electrons observed in mars' ionosphere, Geophysical research letters, 27(13), 1871-1874.

Mitchell, D., R. Lin, C. Mazelle, H. Reme, P. Cloutier, J. Connerney, M. Acun a, and N. Ness (2001), Probing Mars' crustal magnetic field and ionosphere with the MGS electron reflectometer, Journal of Geophysical Research: Planets (1991-2012), 106(E10), 23,419-23,427.

Mitchell, D. L., R. J. Lillis, R. P. Lin, J. E. P. Connerney, and M. H. Acuña (2007), A global map of Mars' crustal magnetic field based on electron reflectometry, J. Geophys. Res., 112, E01002, doi:10.1029/2005JE002564.

Mitchell, D.L., Mazelle, C., Sauvaud, J.A., Thocaven, J.J., Rouzaud, J., Fedorov, A., Rouger, P., Toublanc, D., Taylor, E., Gordon, D. and Robinson, M., 2016. The MAVEN solar wind electron analyzer. Space Science Reviews,200(1-4), pp.495-528.

Morschhauser, A., V. Lesur, and M. Grott (2014), A spherical harmonic model of the lithospheric magnetic field of Mars, J. Geophys. Res. Planets, 119, 1162-1188, doi:10.1002/2013JE004555.

Peterson, W. K., et al. (2016), Photoelectrons and solar ionizing radiation at Mars: Predictions versus MAVEN observations, J. Geophys. Res. Space Physics, 121, doi:10.1002/2016JA022677.

Schneider, Nicholas M., Justin I. Deighan, Sonal K. Jain, Arnaud Stiepen, A. Ian F. Stewart, Davin Larson, David L. Mitchell et al. "Discovery of diffuse aurora on Mars." Science 350, no. 6261 (2015): aad0313. 
Shane, Alexander D., Shaosui Xu, Michael W. Liemohn, and David L. Mitchell (2016). "Mars nightside electrons over strong crustal fields." Journal of Geophysical Research: Space Physics 121, no. 4: 3808-3823.

Steckiewicz, M., C. Mazelle, P. Garnier, N. Andr, E. Penou, A. Beth, J.-A. Sauvaud, D. Toublanc, D. L. Mitchell, J. P. McFadden, J. G. Luhmann, R. J. Lillis, J. E. P. Connerney, J. R. Espley, L. Andersson, J. S. Halekas, D. E. Larson, and B. M. Jakosky (2015), Altitude dependence of nightside Martian Suprathermal electron depletions as revealed by MAVEN observations, Geophysical Research Letters, pp. n/a-n/a, doi: 10.1002/2015GL065257, 2015 GL065257.

Trotignon, J., C. Mazelle, C. Bertucci, and M. Acuna (2006), Martian shock and magnetic pile-up boundary positions and shapes determined from the phobos 2 and mars global surveyor data sets, Planetary and Space Science, 54(4), 357 - 369, doi: http://dx.doi.org/10.1016/j.pss.2006.01.003.

Xu, S., M. W. Liemohn, D. L. Mitchell, and M. D. Smith (2014), Mars photoelectron energy and pitch angle dependence on intense lower atmospheric dust storms, J. Geophys. Res. Planets,119, 1689-1706, doi:10.1002/2013JE004594.

Xu, S., and Liemohn, M. W. (2015), Superthermal electron transport model for Mars. Earth and Space Science, 2, 47-64. doi: 10.1002/2014EA000043.

Xu, S., M. W. Liemohn, and D. L. Mitchell (2015a), Solar wind electron precipitation into the dayside Martian upper atmosphere through the cusps of strong crustal fields, J. Geophys. Res. Space Physics, 119, pages 10,10010,115. doi:10.1002/2014JA020363.

Xu, S., M. W. Liemohn, W. Peterson, J. Fontenla, and P. Chamberlin (2015b), Comparison of different solar irradiance models for the superthermal electron transport model for Mars, Planet. Space Sci., doi:10.1016/j.pss.2015.09.008.

Xu, S., M. Liemohn, S. Bougher, and D. Mitchell (2015c), Enhanced carbon dioxide causing the dust storm-related increase in high-altitude photoelectron fluxes at Mars, Geophys. Res. Lett., 42, 9702-9710, doi:10.1002/2015GL066043.

Xu, S., M. Liemohn, S. Bougher, and D. Mitchell (2016a), Martian high-altitude photoelectrons independent of solar zenith angle, J. Geophys. Res. Space Physics, 121, 3767-3780, doi:10.1002/2015JA022149.

Xu, S. et al., (2016b), Deep nightside photoelectron observations by MAVEN SWEA: implications for Martian northern-hemispheric magnetic topology and nightside ionosphere source, Geophys. Res. Lett., 43, doi:10.1002/2016GL070527. 


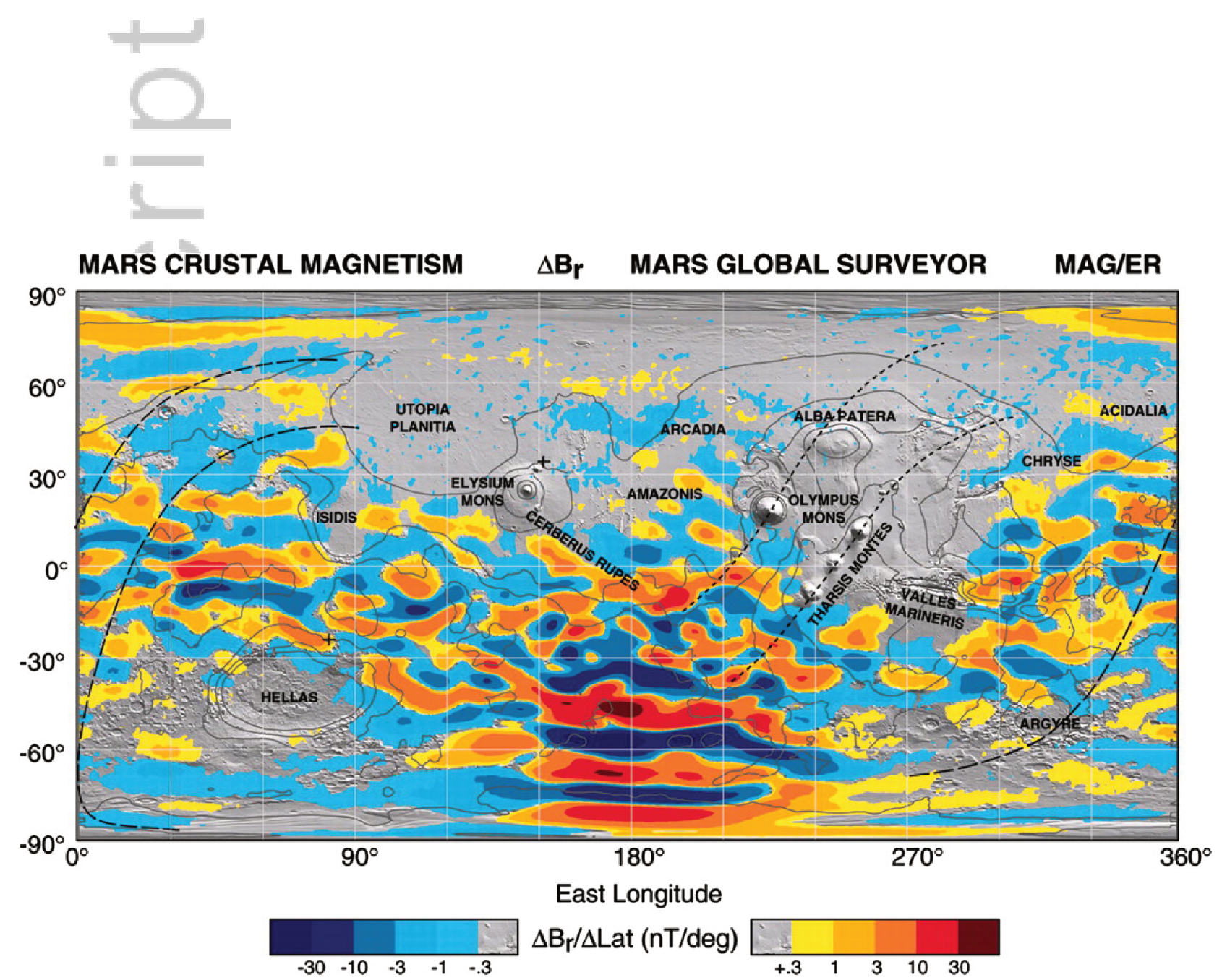

J. E. P. Connerney et al. PNAS 2005; 102:14970-14975

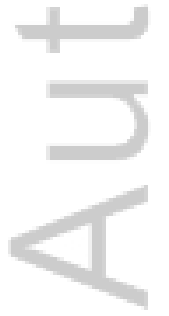

$$
\text { 2016ja023467-f01-z-.eps }
$$

This article is protected by copyright. All rights reserved. 


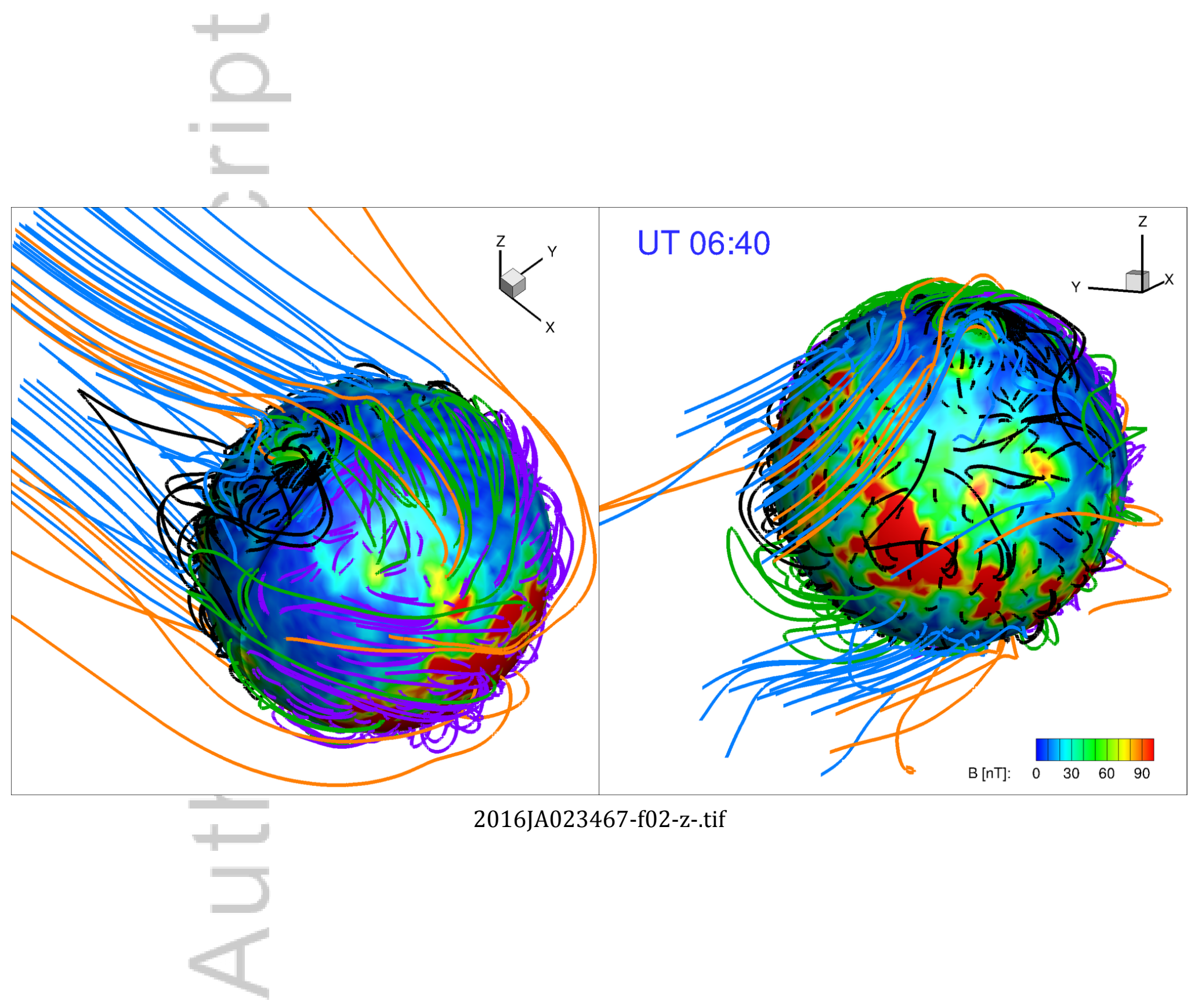

This article is protected by copyright. All rights reserved. 


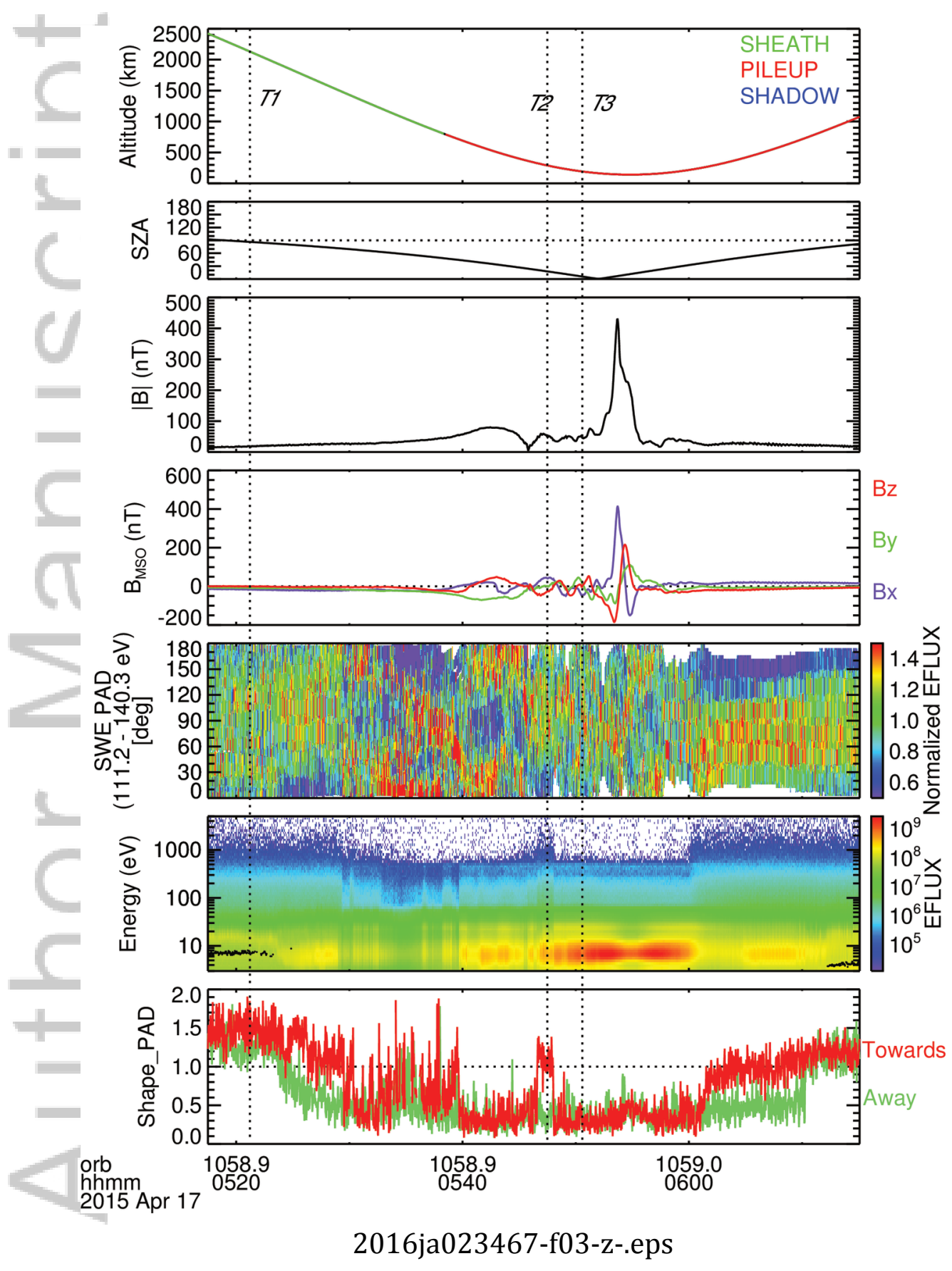

This article is protected by copyright. All rights reserved. 


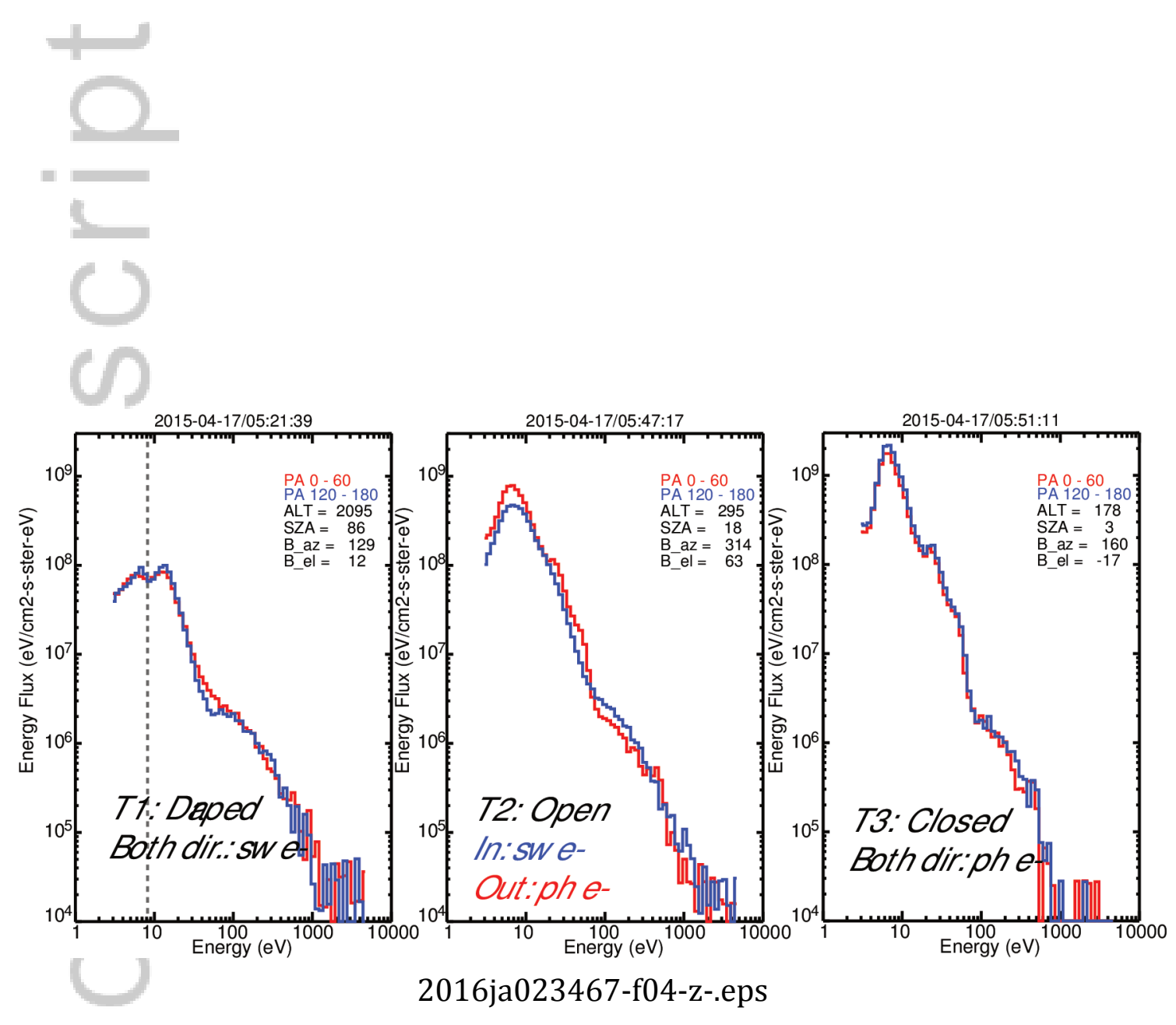

This article is protected by copyright. All rights reserved. 


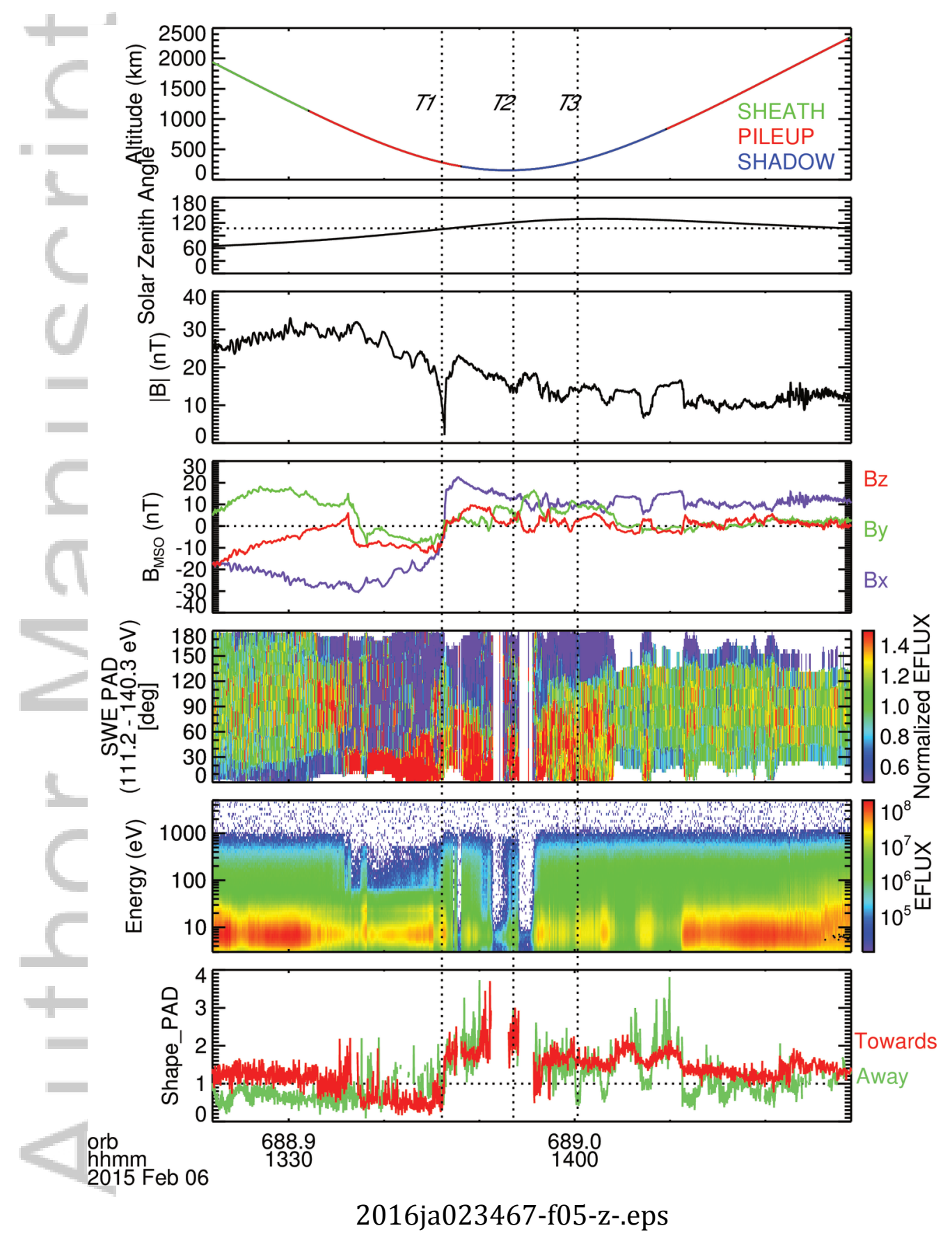

This article is protected by copyright. All rights reserved. 


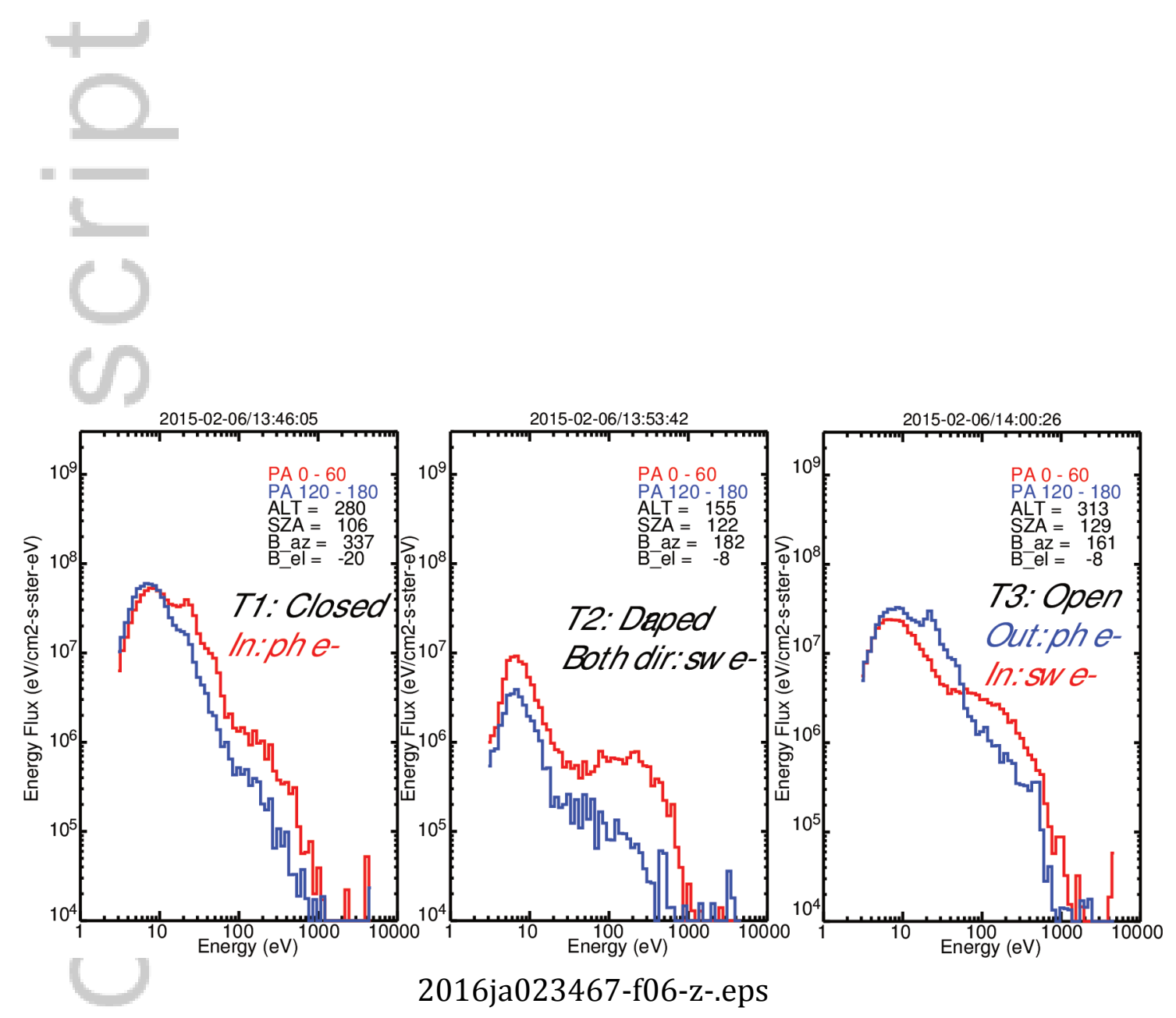

This article is protected by copyright. All rights reserved. 

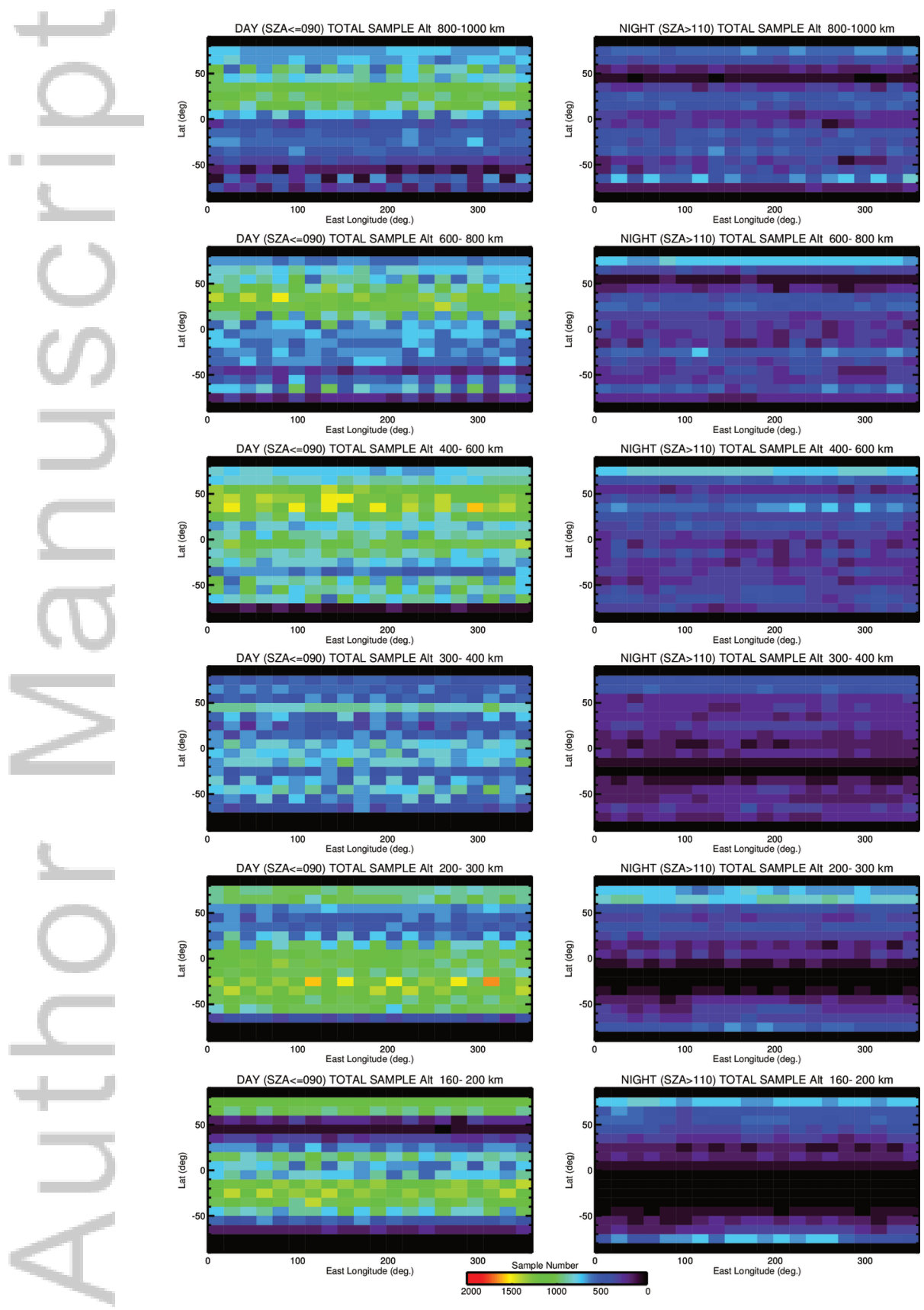

100 East Longitude (deg)

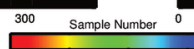

2016ja023467-f07-z-.eps

This article is protected by copyright. All rights reserved. 

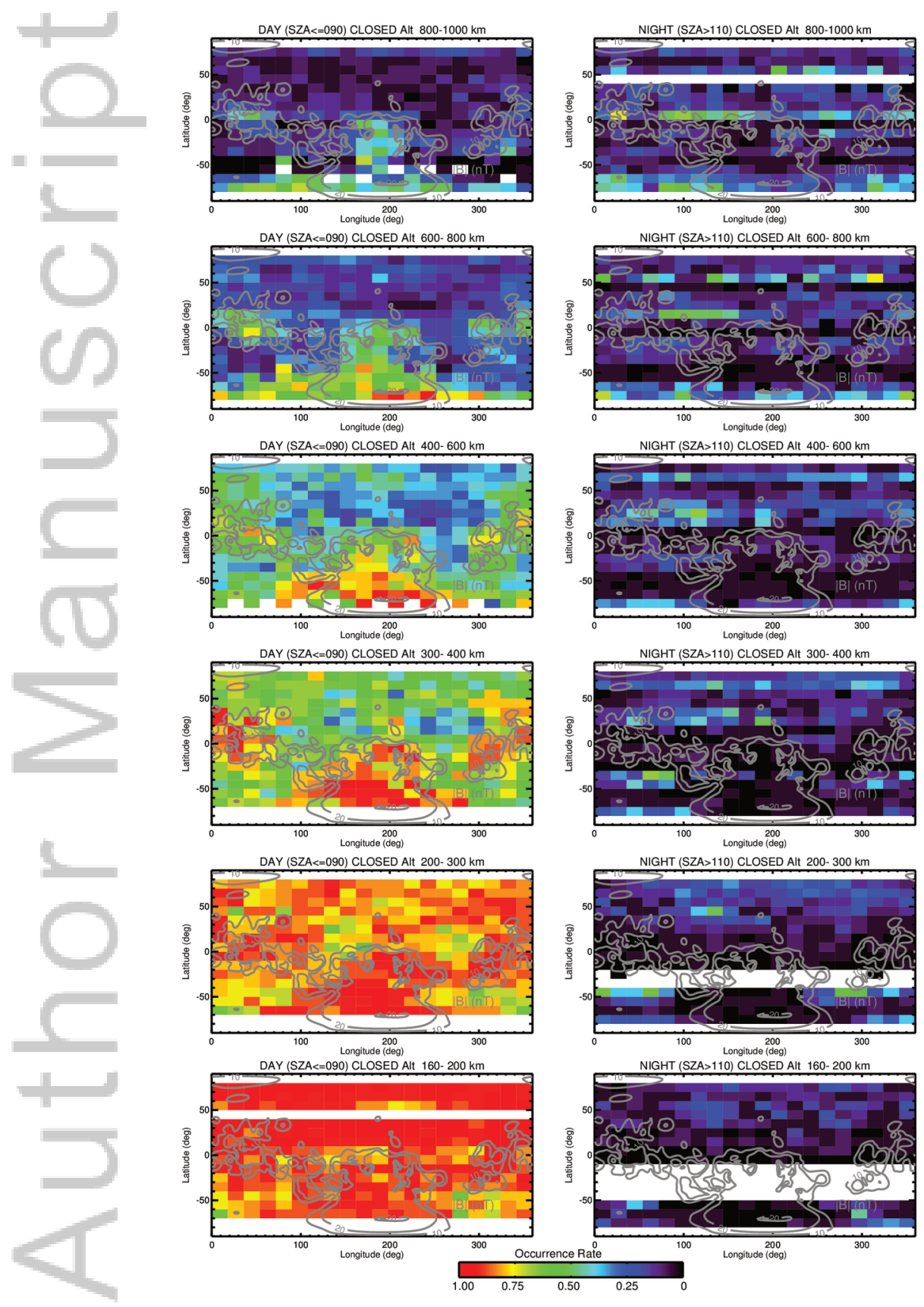

DAY (SZA $c=090$ ) CLOSED Alt $300-400 \mathrm{~km}$
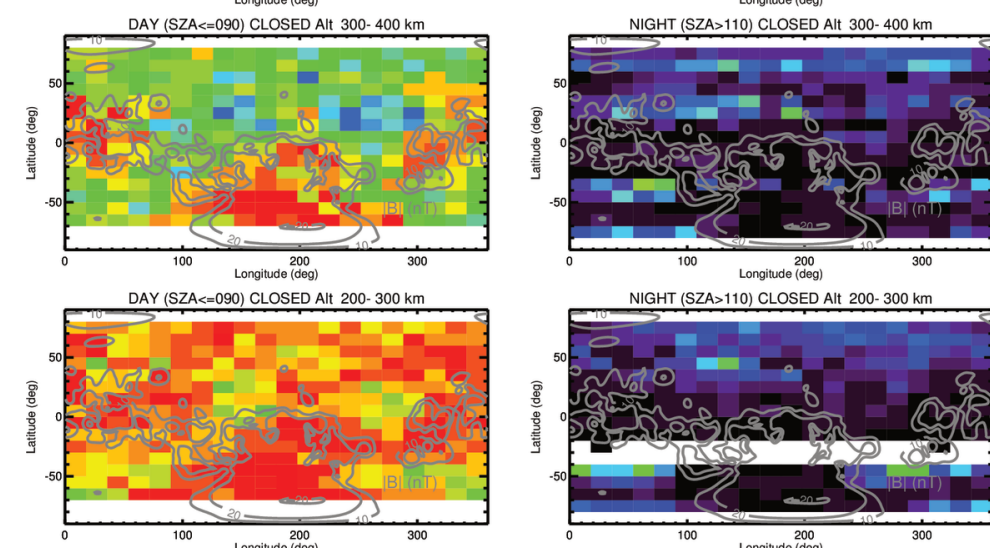

DAY (SZA $<=090$ ) CLOSED Alt $160-200 \mathrm{~km}$

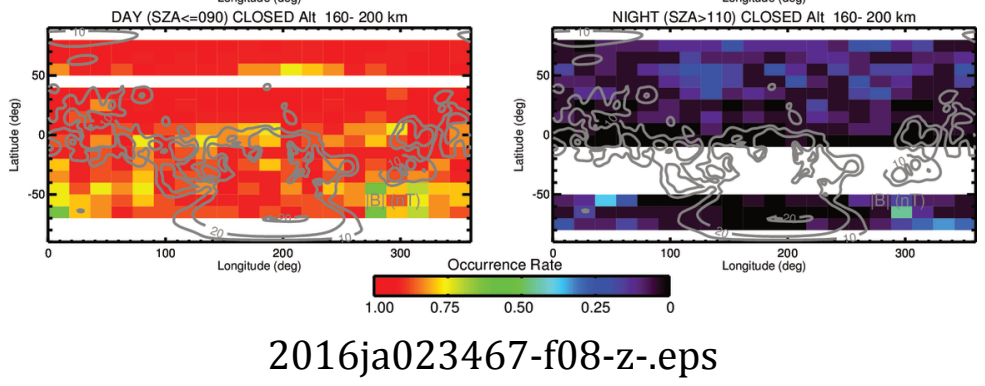

This article is protected by copyright. All rights reserved. 


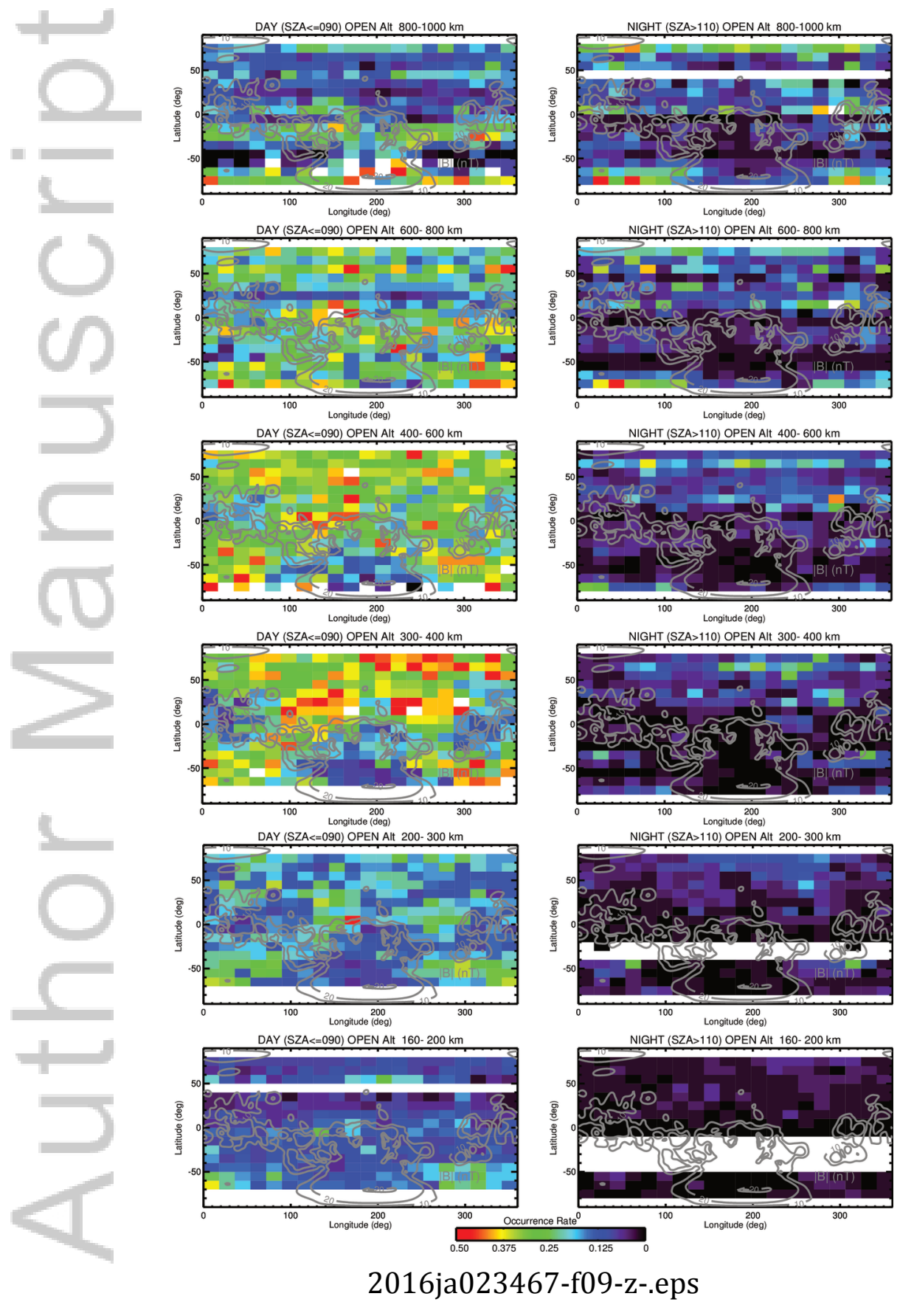

This article is protected by copyright. All rights reserved. 

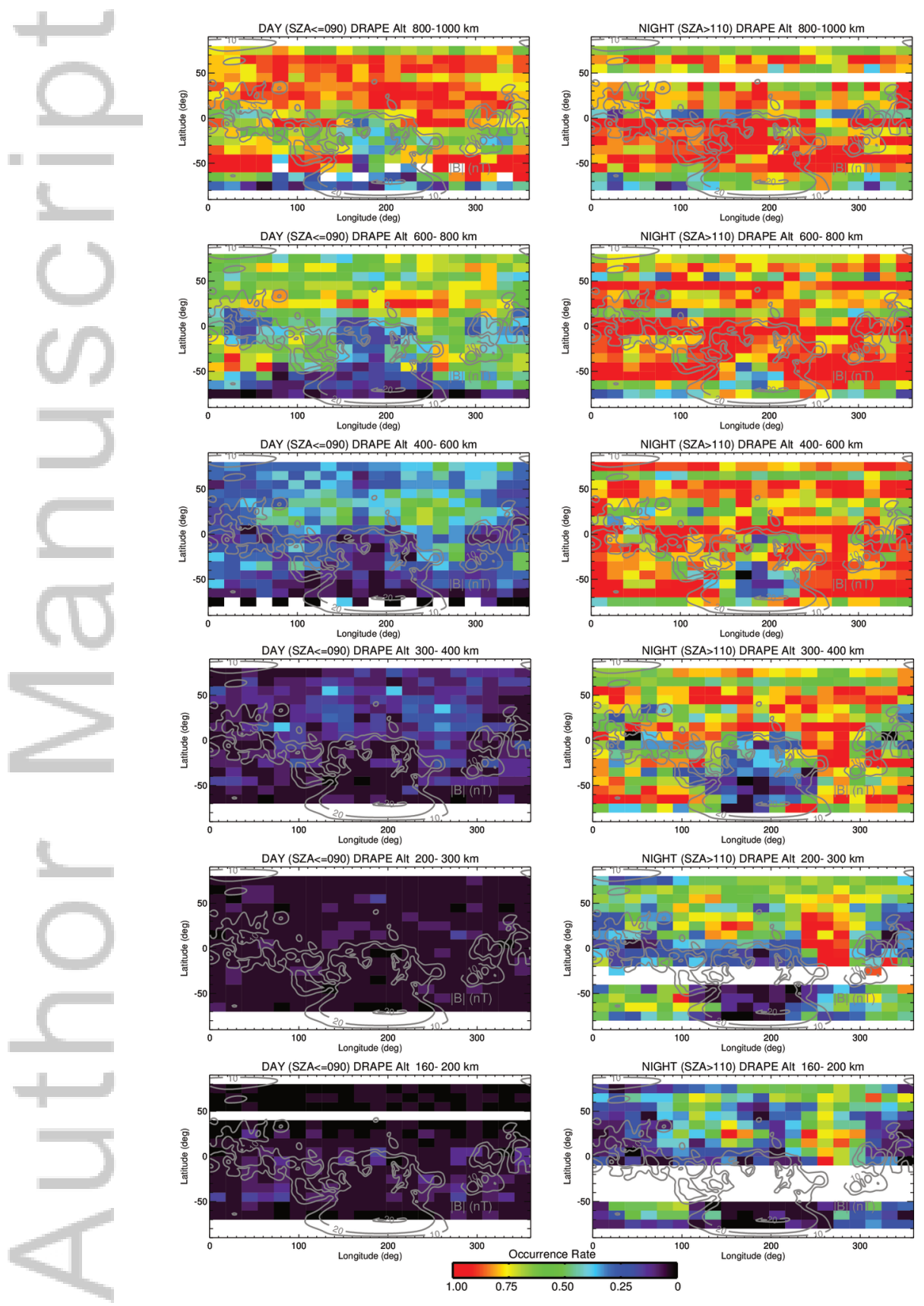

DAY (SZA $<=090)$ DRAPE Alt $160-200 \mathrm{~km}$

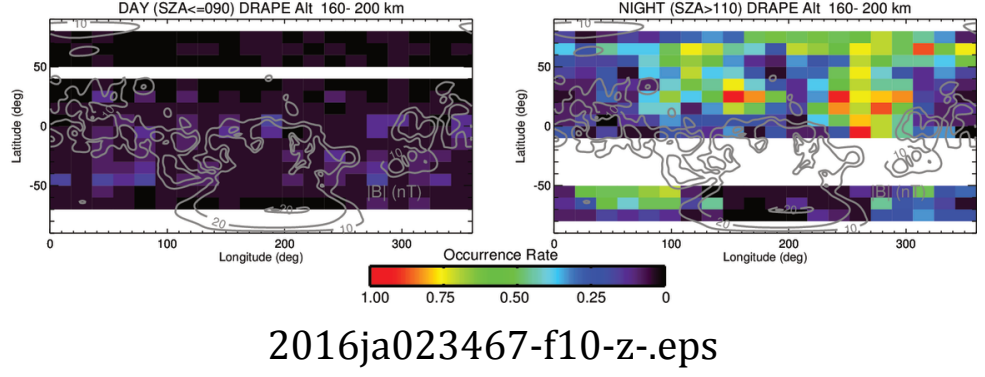

This article is protected by copyright. All rights reserved. 

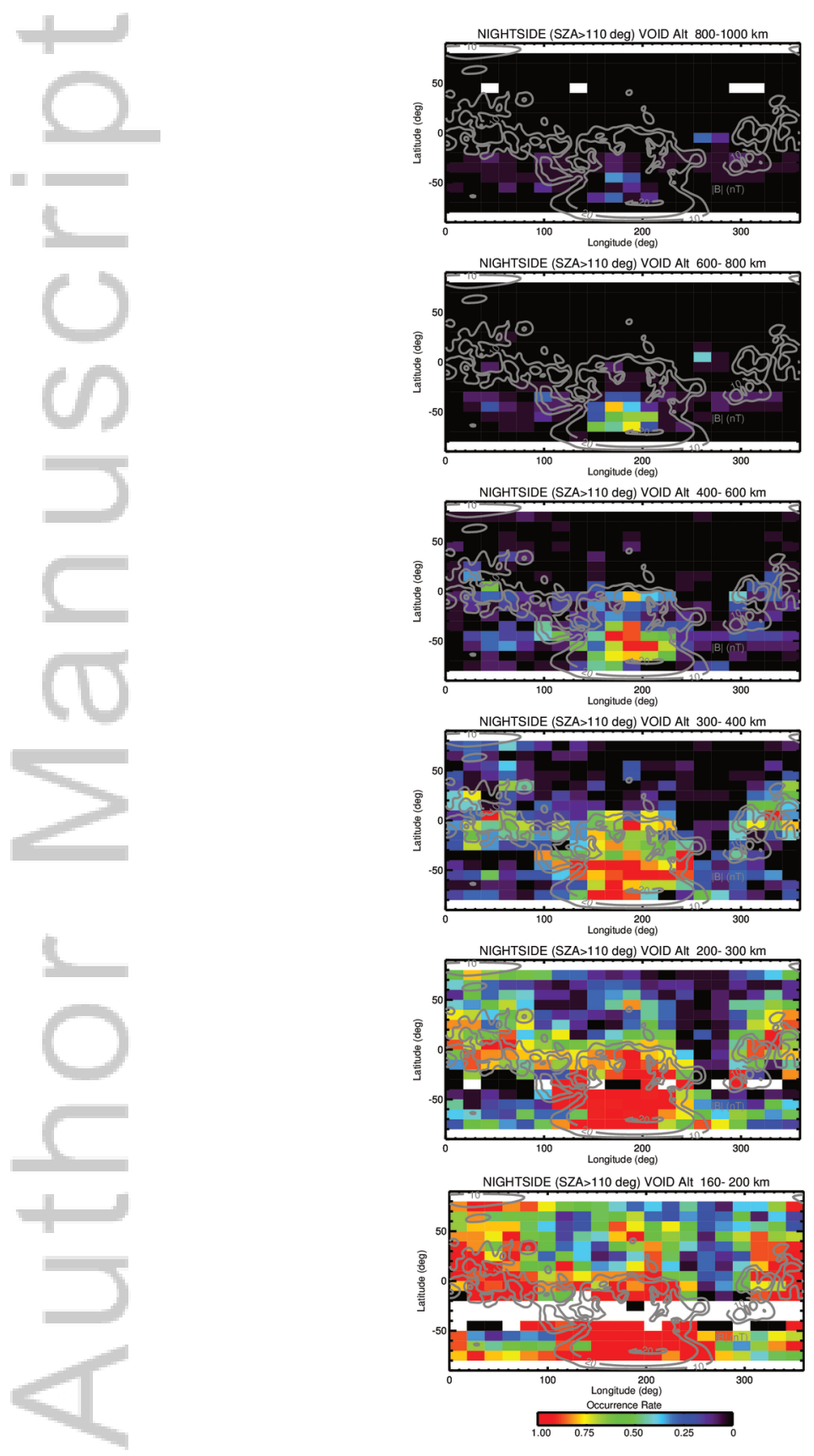

2016ja023467-f11-z-.eps

This article is protected by copyright. All rights reserved. 


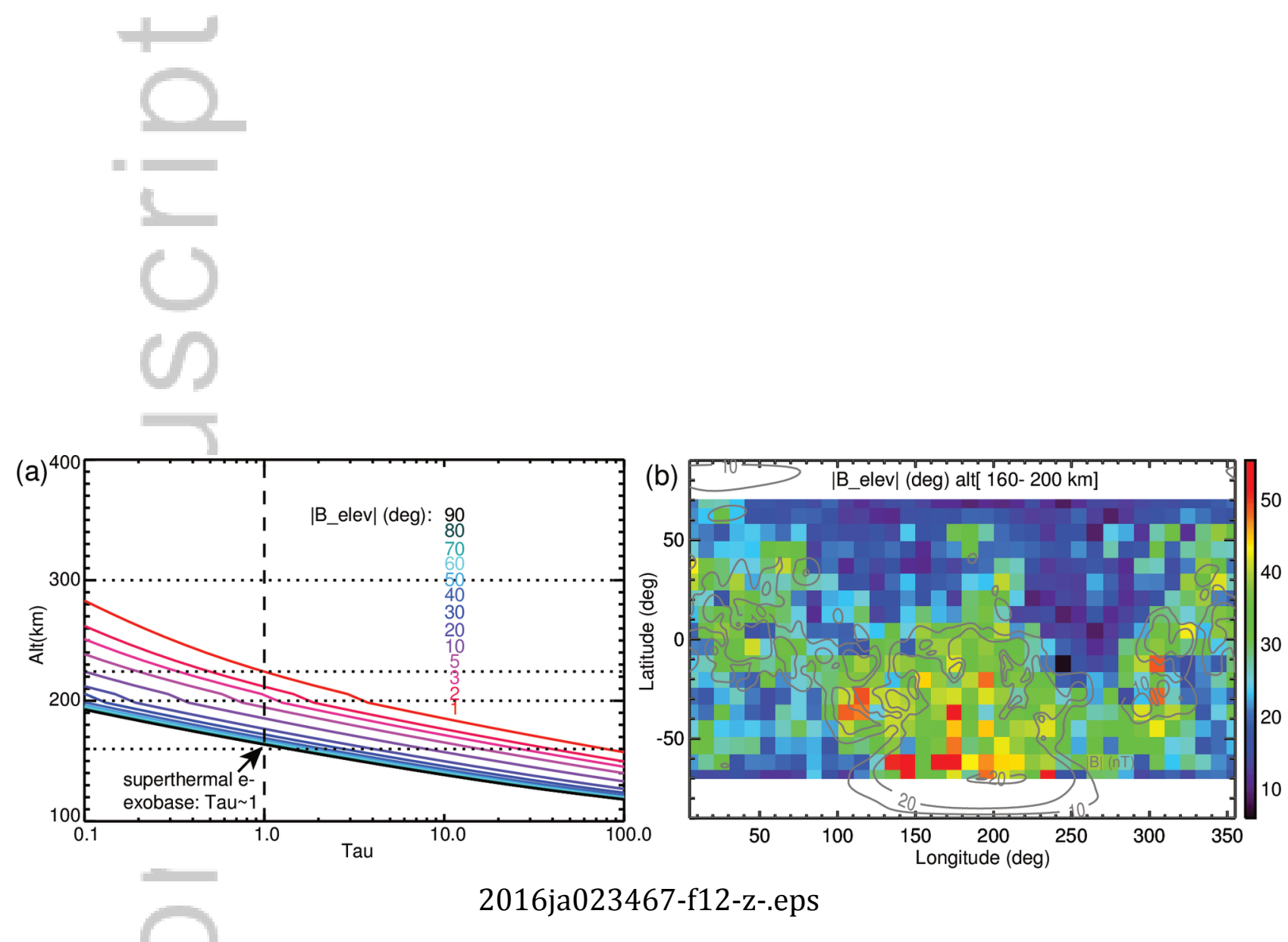

This article is protected by copyright. All rights reserved. 